This article was downloaded by:[Almonacid, Pablo Mata]

On: 26 June 2007

[Almonacid, Pablo Mata]

Access Details: [subscription number 779897676]

Publisher: Taylor \& Francis

Informa Ltd Registered in England and Wales Registered Number: 1072954

Registered office: Mortimer House, 37-41 Mortimer Street, London W1T 3JH, UK

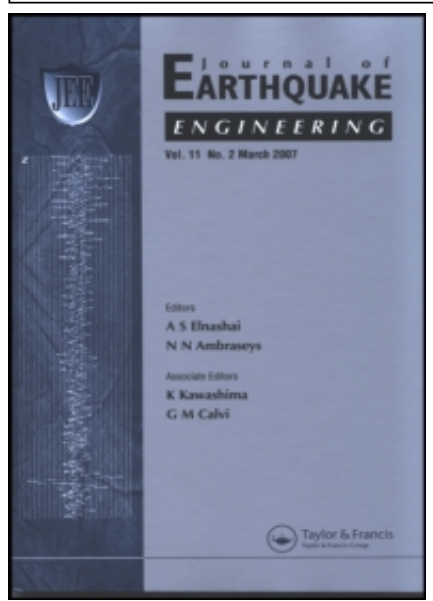

Journal of Earthquake Engineering

Publication details, including instructions for authors and subscription information: http://www.informaworld.com/smpp/title content=t741771161

High Damping Rubber Model for Energy Dissipating Devices

To cite this Article: Mata, Pablo, Boroschek, Ruben, Barbat, Alex H. and Oller, Sergio , 'High Damping Rubber Model for Energy Dissipating Devices', Journal of Earthquake Engineering, 11:2, 231 - 256

To link to this article: DOI: $10.1080 / 13632460601123214$

URL: http://dx.doi.org/10.1080/13632460601123214

Full terms and conditions of use: http://www.informaworld.com/terms-and-conditions-of-access.pdf

This article maybe used for research, teaching and private study purposes. Any substantial or systematic reproduction, re-distribution, re-selling, loan or sub-licensing, systematic supply or distribution in any form to anyone is expressly forbidden.

The publisher does not give any warranty express or implied or make any representation that the contents will be complete or accurate or up to date. The accuracy of any instructions, formulae and drug doses should be independently verified with primary sources. The publisher shall not be liable for any loss, actions, claims, proceedings, demand or costs or damages whatsoever or howsoever caused arising directly or indirectly in connection with or arising out of the use of this material.

(C) Taylor and Francis 2007 


\title{
High Damping Rubber Model for Energy Dissipating Devices
}

\author{
PABLO MATA \\ Technical University of Catalonia, Barcelona, Spain
}

RUBEN BOROSCHEK

University of Chile, Civil Engineering Department, Santiago, Chile

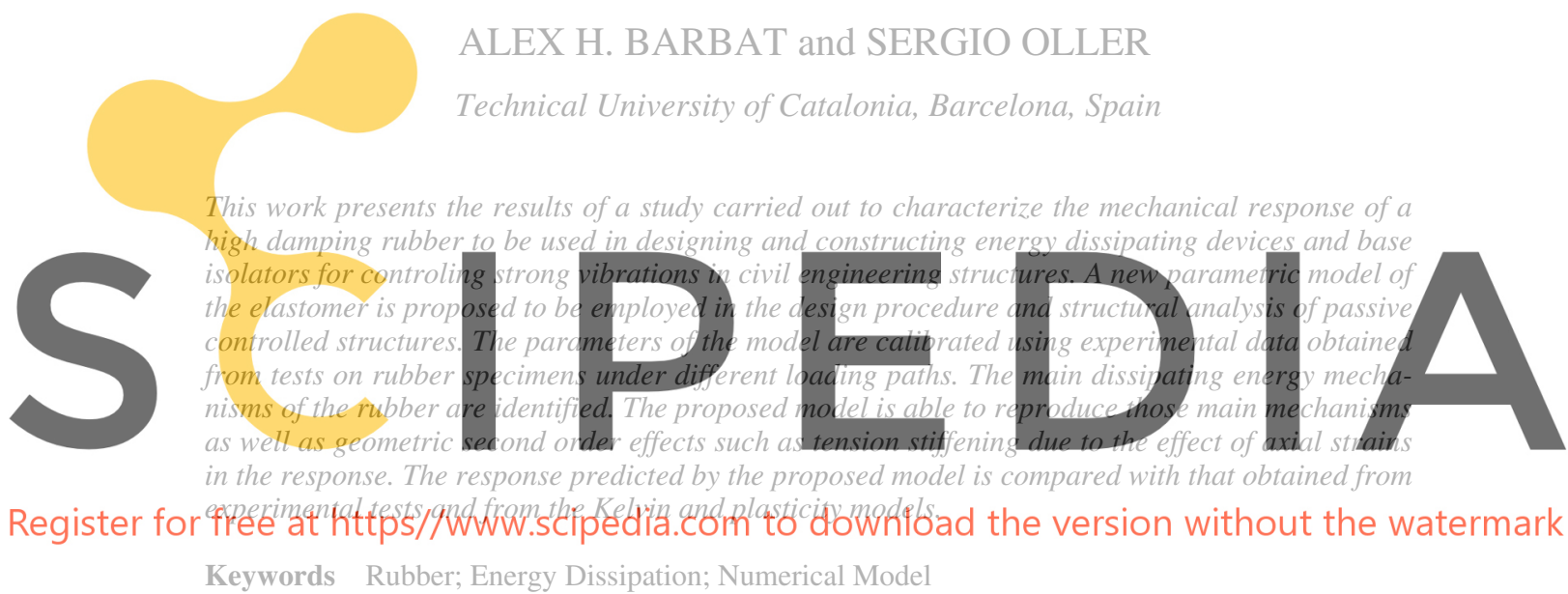

\section{Introduction}

Rubber is one of the most frequently used materials in the construction of base isolation systems [Barbat and Bozzo, 1997; Calvi and Pavese, 1997; Bixio et al., 2001,] and energy dissipating devices for controling vibrations induced by earthquakes, machineries, pedestrian loads, and wind. The mechanical characteristics of rubber, as well as its energy dissipating capacity, depends strongly on the composition of the mixture used and on the thermo chemical processes followed in the fabrication of the material. In general, its mechanical behavior is complex showing an elastic modulus strongly dependent on the strain level, temperature and loading frequency [Kojima and Yoshihide, 1990; AASHTO, 1991; Ahmadi and Muhr, 1997]. Due to these characteristics, rubber shows different types of energy dissipating mechanisms, which can go from purely hysteretic to viscous or others [Hwang et al., 1997]. Additionally, when rubber undergoes large strains, and depending on the boundary conditions, a noticeable hardening phenomenon can appears in the strain-stress

Received 19 September 2005; accepted 16 June 2006.

Address correspondence to Pablo Mata, Technical University of Catalonia, Edificio C1, Campus Norte UPC. Gran Capitán s/n, Barcelona 08034; E-mail: pmata@cimne.upc.edu 
response [Kelly, 1988; Mata and Boroschek, 2001]. Because of these reasons, it is not usual to find numerical models describing in a suitable way the response of this kind of materials when subjected to displacements involving large strains.

An appropriated constitutive description for high damping rubbers should consider large displacements, nonlinear strain-displacement relationship, incompressibility of the material, and its dissipative characteristics [Asano et al., 2000; Soong and Dargush, 1997]. Nowadays, it is possible to find numerical models of the problem based on the principles of the continuous solid mechanics with appropriate constitutive laws for the components of the rubber, but normally they require a large amount of computer time and the convergence of the problem is not ensured [Salomon et al., 1999; Belytschko et al., 2000].

Other kind of models can be obtained by fitting the parameters of equations or algorithms to the curves obtained experimentally [Sues et al., 1988; Dusi et al., 1999; Naeim and Kelly, 1999], providing a description of the material behavior. This last choice has the advantage of being much less demanding in computational time and it is possible to calibrate the model directly from loading tests carried out on specimens, although the provided model is able only to describe the behavior of the specimen for the boundary conditions of the tests.

Frequently, the practical design of structures with rubber based control systems employs equivalent linear models for the material of the controlling devices [Kelly, 1988; Moroni et al., 1988]. One example of these are the specifications given by the American Association of State Highway and Transportation Officials, [AASHTO, 1991]. The use of equivalent linear models can induce significant errors [Ahmadi and Muhr, 1997; Dusi

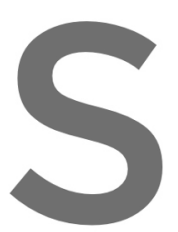
et al., 1999; Mata et al., 2004] in the estimation of the ene
even when some times the peak structural response, 1.e., d
can be estimated with a reasonably level of accuracy for
employing equivalent inear models and an iterative checking p
1997]. On the contrary, if we are interested in a more detailed
have to consider thatequivalent linear models represent a so
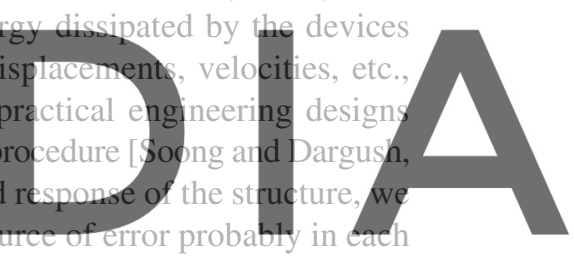

time step due to the fact that the values for the elastic modulus and damping coefficient

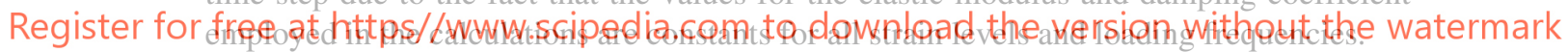

This work develops an analytical model for the strain-stress relationship of a high

damping rubber employed in energy dissipating devices. The proposed model is an extension of the plasticity model proposed by Wilson [1998], and implemented in the software package SAP2000 [SAP, 2004]. The proposed extension shows ability in simulating the axial hardening phenomenon in large strains as well as a variable shear modulus. The model depends on a set of parameters that are calibrated from data obtained with experimental tests. Even when the number of employed parameters is greater than in other models [see, e.g., Dusi et al., 1999], the proposed model avoids the necessity of work with parallel elements for simulating variable shear modulus and, additionally, it takes into account non linear viscosity, what allows to simulate a wide variety of rubber types [Kojima and Yoshihide, 1990]. The response of the model for arbitrary loading paths is compared with experimental data and with the responses obtained from Kelvin's and plasticity models.

\section{Experimental Characterization of High Damping Rubber Specimens}

The force-displacement response of the high damping rubber was obtained for different loading paths, which include sinusoidal cycles of imposed displacements with different amplitudes and frequencies. Sequences of arbitrary imposed displacements were also employed. The variations of the mechanical properties of the rubber with temperature, aging, or environmental effects were not studied in this part of the work. However, dependency between dissipated energy and loading frequency or maximum strain level was 
studied. An identification of two dissipating mechanisms is proposed, classifying them in non viscous and viscous according to their dependency on displacements or velocities respectively, together with a method to set out an equivalent viscous damping coefficient [Sarrazín et al., 1993; Ponce León et al., 1997].

\subsection{Description of the Experimental Setup and Test Program}

Three specimens were tested for obtaining the mechanical characterization of the rubber. Each test specimen is composed of five segments: both extreme segments and the central one are made of steel with circular perforations for providing the fixation of the specimen to the test machine, while the other two are rubber plates of $900 \mathrm{~mm}^{2}$ of area and $6 \mathrm{~mm}$ of thickness. The faces of the rubber plates were adhered to the contiguous steel bodies. During the tests, the specimens were restricted to maintain zero relative movement in the direction perpendicular to the imposed displacement, as it can be seen in Fig. 1a. By this way, fixing the extreme segments and forcing a displacement in the intermediate one, it is possible to develop different loading paths of imposed displacement. The central embolus is connected to the hydraulic actuator of the test machine which is servo mechanically controlled by means of a computer program. For each applied displacement, it is possible to determine the average shear strain, $\varepsilon$, and the average shear stress, $\sigma$, in a rubber plate by means of the formulas [Naeim and Kelly, 1999],
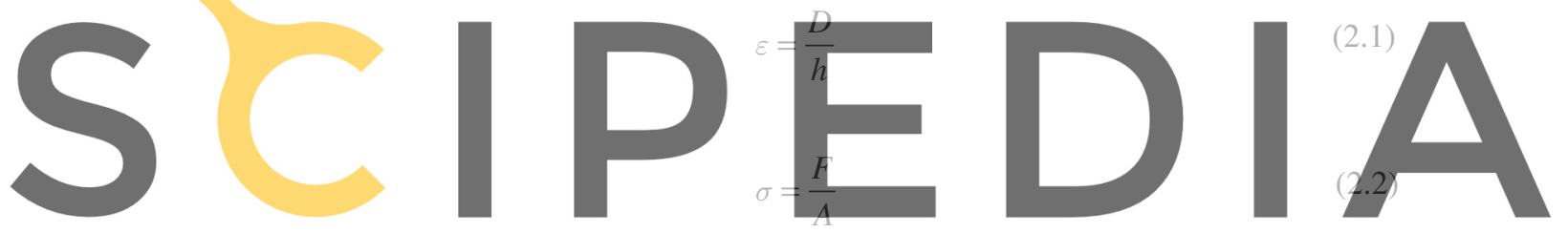

Register for free at https//www.scipediascom to download the version without the watermark

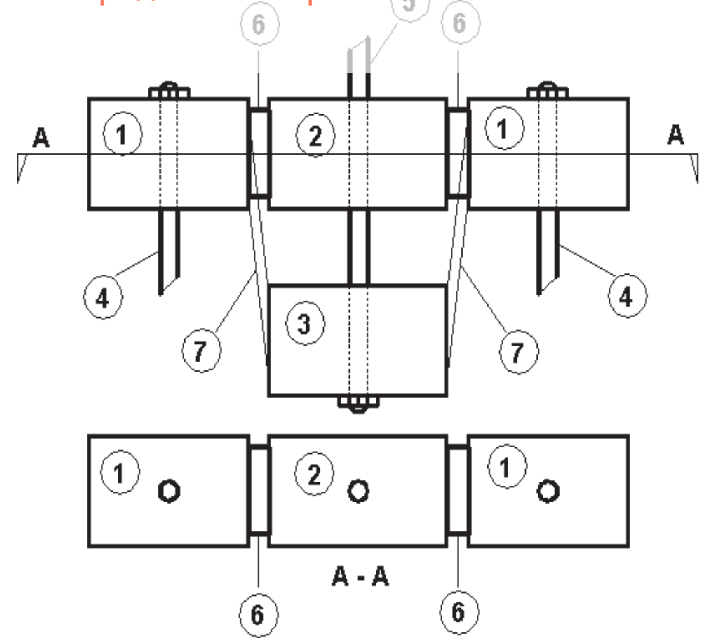

a

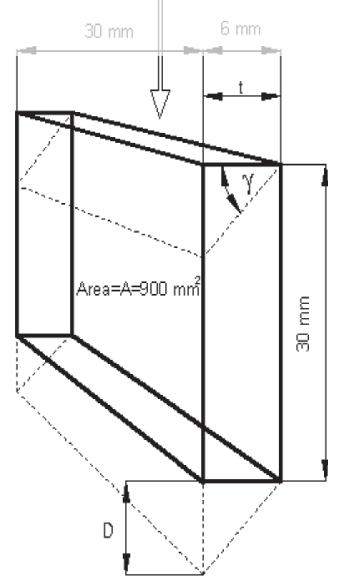

b

FIGURE 1 (a) Test specimen description. 1: Fixed steel segments. 2: Movable steel segment. 3: Position of the movable steel segments when a displacement is imposed on the specimen. 4: Fixing bolts. 5: Movable embolus. 6: Rubber plate in original configuration. 7: Deformed rubber specimen. (b) Detail of the rubber plate in deformed position. 
where $D$ is the applied displacement, $h$ is the plate thickness, $A$ is the plate area, and $F$ is the force necessary to obtain $D$. In Fig. $1 b$ it is possible to see a diagram where appears these quantities. The sequence of loading tests was the following:

- Symmetric cycles. The three specimens were subjected to sinusoidal cycles of imposed displacements with frequencies $1 / 30,0,5,1,0$, and $2,0 \mathrm{~Hz}$, producing the following maximum shear strains: $+/-10,20,50,100,150,170$, and $200 \%$. By means of this set of tests, hysteretic cycles in the rubber specimens were obtained.

- Asymmetric cycles. Sinusoidal cycles of imposed displacement with different maximum and minimum shear strains were studied. The tests were carried out for loading frequency of $0,5 \mathrm{~Hz}$. In Table 1 , detailed values of the applied maximum and minimum shear strains are given. As an example, it can be seen in the cyclic test 1 of Table 1 , the specimen is deformed to reach a maximum strain level of $200 \%$ and then unloaded until a strain level of $170 \%$.

- Arbitrary imposed displacements. In this case, the specimens were subjected to two sequences of arbitrary imposed displacements, with loading velocity of 133 and 400 loading steps per second, respectively. Figs. $2 \mathrm{a}$ and $2 \mathrm{~b}$ show the shape of the employed records. The first one corresponds to a sequence of ramps applied for inducing controlled hysteretic cycles, and the second one corresponds to the scaled displacement response of a single degree of freedom system with a period of $1.0 \mathrm{~s}$ and $5 \%$ of critical damping subjected to the N-S component of the El Centro, 1940,

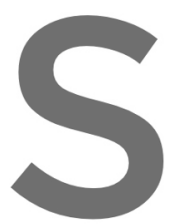
earthquake. The strain of $200 \%$ in the the rubber response seismic case. Figs, $2 \mathrm{c}$ and $2 \mathrm{c}$
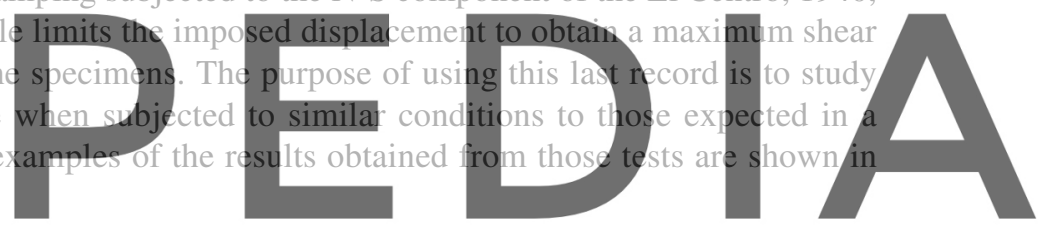

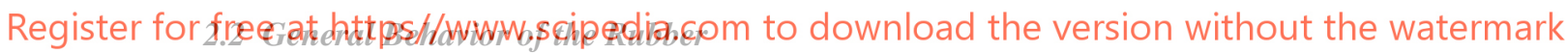

The general behavior of the rubber can be described as hysteretic, showing a nonlinear strain-stress relationship, with energy dissipation for any maximum strain level and loading frequency.

When a test specimen is subjected for the first time to sinusoidal cycles of imposed displacements, it is possible to observe that the material suffers progressive stiffness degradation, as it can be seen in Fig. 3a. The degrading process progresses until a stable shape of the hysteretic cycle is obtained. Approximately five cycles are required to stabilize the degrading process. This progressive lack of stiffness could be explained as a transient process of rearrangement of the particles, which compose the material. The rubber carries out this process to withstand a new state of loadings given by the shear strain associated with de imposed displacements [Salomon et al., 1999]. When a new internal configuration, compatible with the environment is reached, the degrading process stops and the material reaches a stable hysteretic state as it is shown in Fig. 3b. In this work, only stabilized hysteretic cycles are characterized.

One of the main characteristics of the rubber noticeable from the tests is a strongly variable shear modulus in any range of strain level and loading frequency of the experiments. It is possible to see in Fig. 4 that, for any point of a loading or unloading branch, the slope of the tangent to the curve is different. However, when the response of the material to symmetric loading cycles are represented in the same graphic, it is possible to identify the following three different zones: 


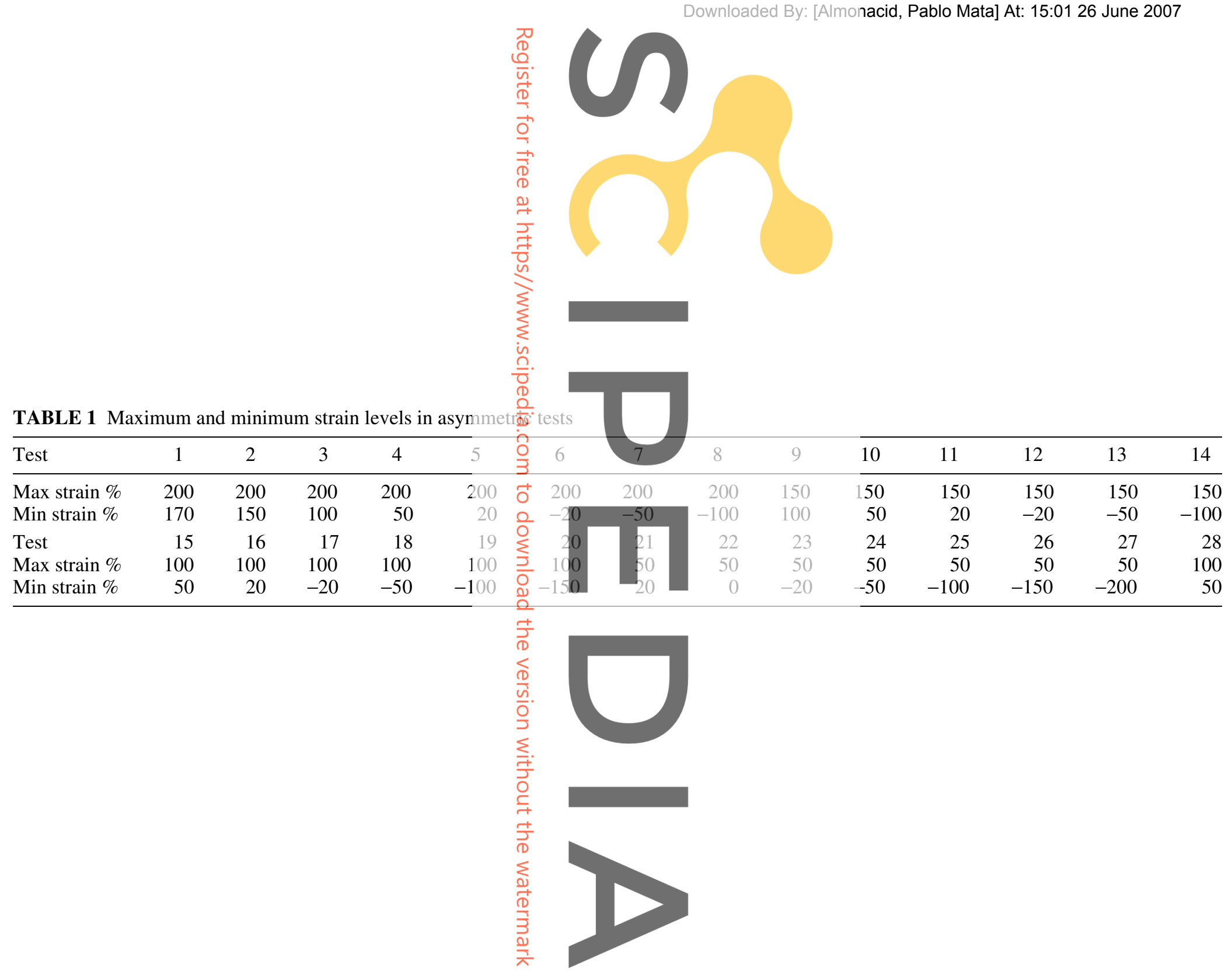




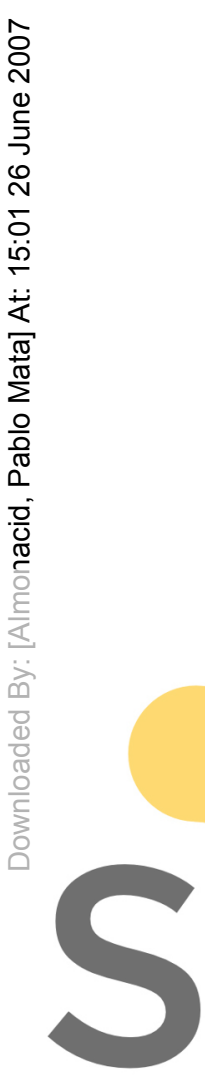

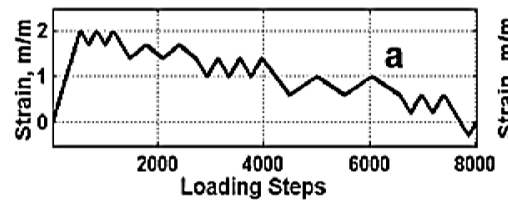

Hysteretic cycles, Secuence of ramps

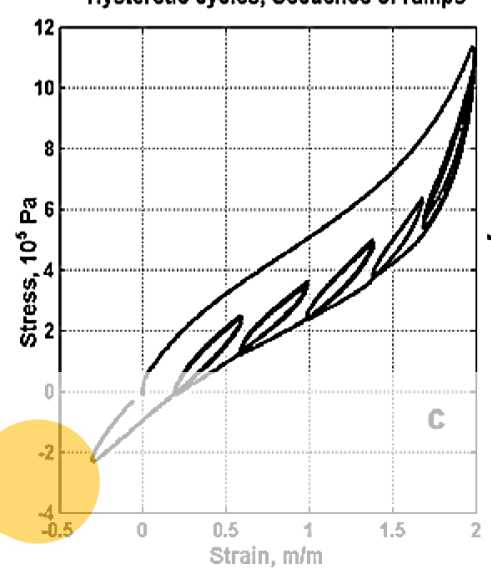

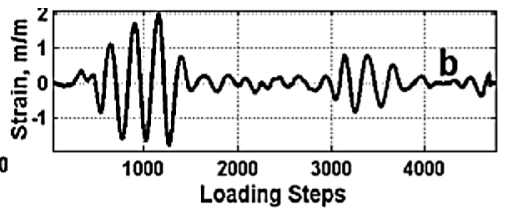

Hysteretic cycles, Earthquake record

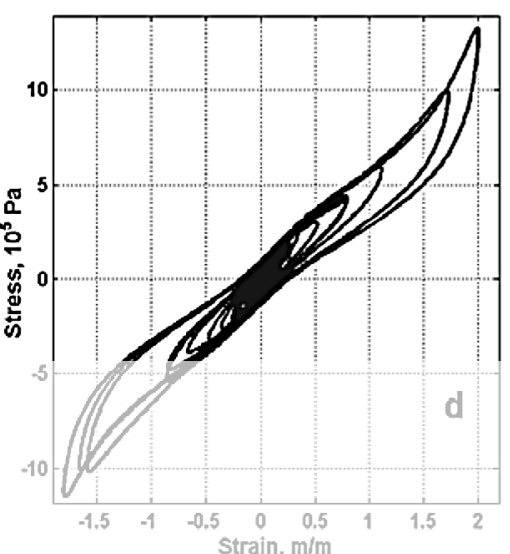

FIGURE 2 Arbitrary records. (a) Sequence of ramps. (b) Scaled displacement response obtained from a linear SDOF system subjected to the N-S component of the El Centro, 1940 earthquake record. (c) Hystere tic cycle obtained for the sequence of ranps. (d) Hysteretic
cycles for the displacement response of a SDOE.

Register for free at https//Wæww.scipedia.com/to dowhload the version without the watermark
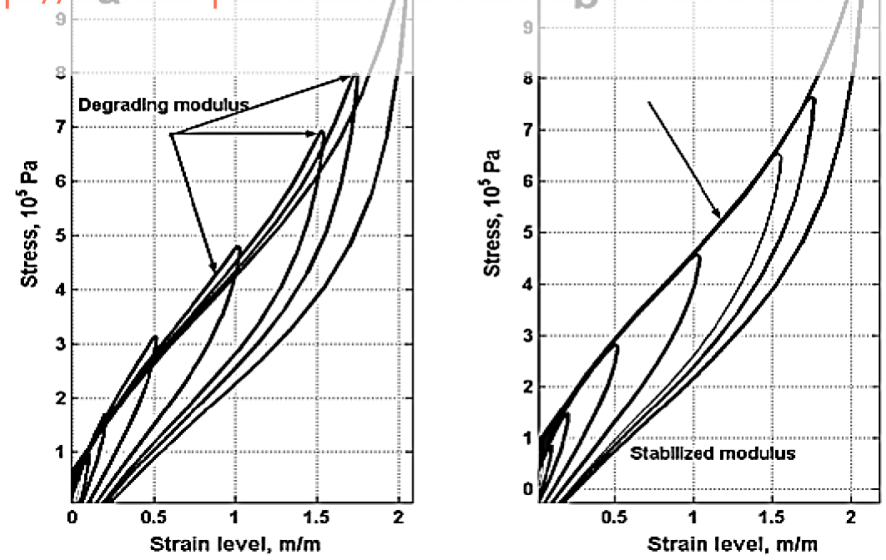

FIGURE 3 (a) Progressive stiffness degradation for repeated loadings cycles. (b) Stabilized cycles.

- Initial Zone 1 in Fig. 4 for strains in the range $+/-20 \%$. This zone is characterized by a high and approximately constant slope of the tangent to the strain-stress curve.

- Zone 2 in Fig. 4, corresponding to the range +/-[20-150] \%, where the slope of the tangent decreases. 
General Behavior of the Rubber

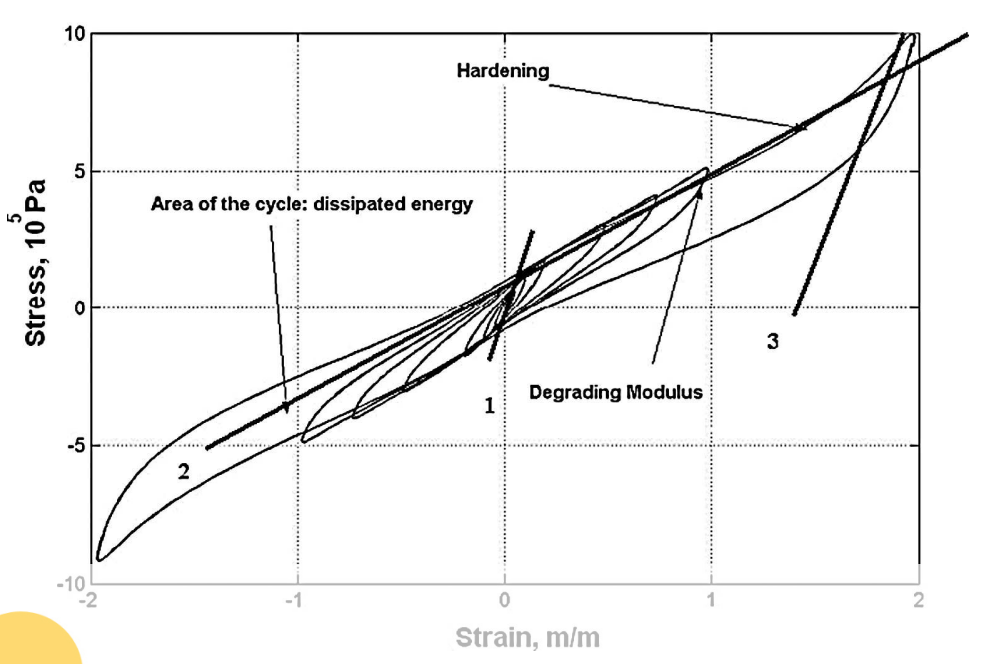

FIGURE 4 Response to symmetric loading. It is possible to appreciate three zones corresponding to the average slopes of the tangent to the strain-stress curves.
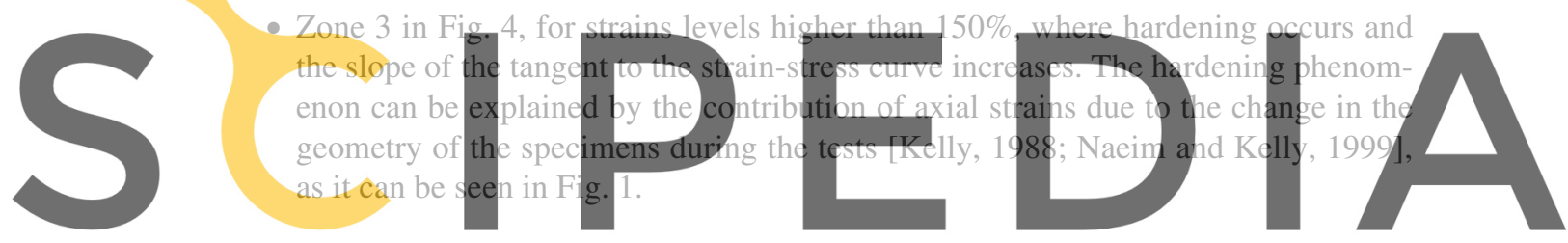

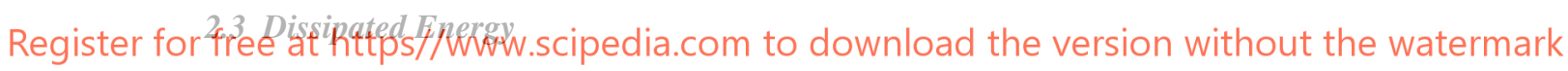

Energy dissipation in rubber-like materials involves internal friction between the particles

of the mixture, viscous damping, and micro zones where plasticity or damage can occur, among others physical changes [Sommer, 1989]. In this work, the total dissipated energy in each loading cycle was calculated estimating the area of the hysteretic cycle [Barbat et al., 1997; Chopra, 2000] independently of the nature of the micro mechanisms involved in the dissipative process.

2.3.1 Dependency Between the Dissipated Energy and the Strain Level. Figure 5 shows the values for the dissipated energy as a function of the maximum strain level reached during symmetric tests for different loading frequencies. As could be expected, the dissipated energy increases with the maximum strain level [Naeim and Kelly, 1999; Mata et al., 2004], when higher displacements are imposed, the test specimen undergoes larger nonlinear strains and, therefore, more energy is dissipated. However, the rate of energy dissipation with the strain level is not constant, which would be the case if a straight line would have been obtained in Fig. 5. The shape of the obtained curves is related with the change of shape of the hysteretic cycles. For low strain levels, the cycles have approximately an elliptic shape, like in Fig. 4, but when the strains are increased, hysteretic cycles are stretched along the major axis of the ellipse, without a significant enlargement along minor axis, in agreement with the results obtained by Ahmadi and Muhr [1997]. This behavior suggests that Kelvin's model would not be a good choice for modeling the rubber behavior in large strains. Finally, for strain levels over $150 \%$, axial hardening appears in the response. 


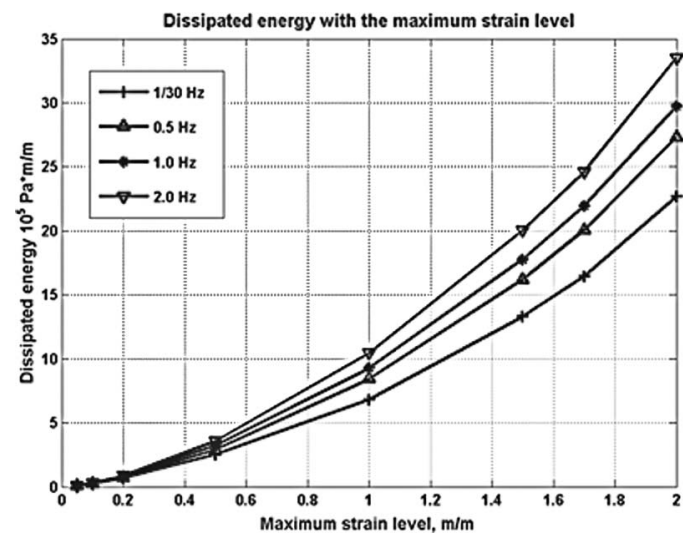

FIGURE 5 Dependency between the dissipated energy and the maximum strain level. Each curve correspond to a fixed value of loading frequency.

2.3.2 Dependency Between Dissipated Energy and Loading Frequency. Figure 6 shows the dependency between dissipated energy and loading frequency. Dissipation increases with frequency. It indicates that stress increments are associated with frequency increments and therefore, forces of viscous nature appears in the rubber response. The results of this Figure are normalized to the value $1 \mathrm{for}$ the test carried out at 1,33Hz, and for each maximum strain level. Addition: $200 \%$, the bigger the pation is maintained Fig. 6, a fact that is evidenced by the approximately constant slope of these curves. range of frequencies $1 / 3$
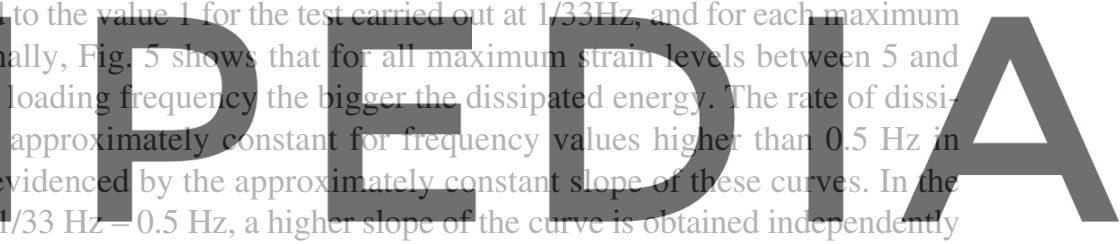
of the strain level. A possible explanation for this phenomenon is given by the existence of

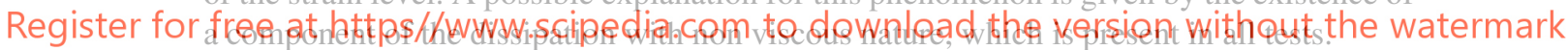

A starting hypothesis of this work is that for cuasi static loadings whit $1 / 33 \mathrm{~Hz}$, dissipation is a function of only the maximum strain level; for higher frequencies and constant strain levels, the energy dissipation would be produced by viscous mechanisms added to

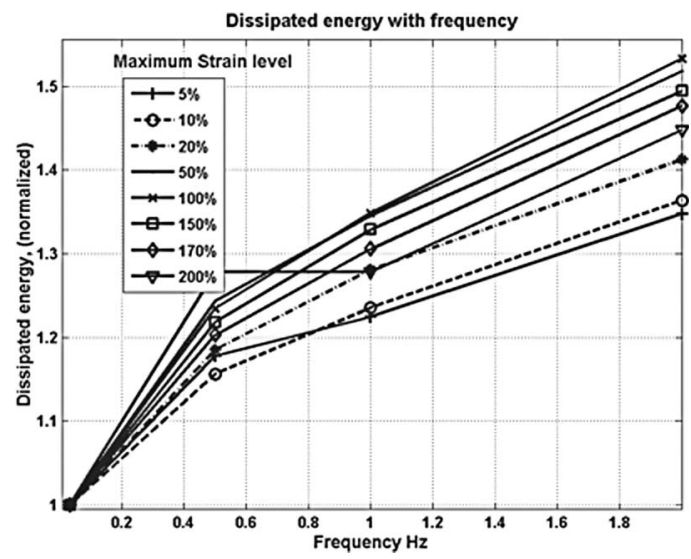

FIGURE 6 Dissipated energy normalized to 1 as a function of the loading frequency. Each curve correspond to a maximum strain level. 
the non viscous ones [Ahmadi and Muhr, 1997]. Figure 7 shows the experimental evidence of the proposed dissipation mechanisms, for two loading tests carried out at $10 \%$ and $200 \%$ of maximum strain level, the area of the hysteretic cycles increases with the frequency.

\subsection{Characterization of the Non-viscous Component of the Response}

As mentioned previously, it is possible to appreciate non-viscous hysteresis in low frequency tests $(1 / 33 \mathrm{~Hz})$. Additionally, it can be observed from the hysteretic cycles obtained from asymmetric loadings that the material follows a reloading or unloading path, which is dependent on the point where the velocity change of sign. It is possible to see in Fig. 4 that the loading branches (velocity of deformation $>0$ ) converge to the enveloping curve of all strain-stress curves. Additionally, it is possible to distinguish that, independently on the unloading path, all curves converge tangentially to a common point located on the lower enveloping curve corresponding to the $10 \%$ of strain level.

\subsubsection{Rubber Behavior to Asymmetric Loading Cycles. In Fig 8a, pointed lines show all} the hysteretic cycles for different minimum strain levels and maximum strain of $200 \%$. In Figs. $8 \mathrm{~b}$ and $8 \mathrm{~d}$, the maximum strains are $150 \%$ and $100 \%$, respectively. In all of the cases, the cycle corresponding to the symmetrical sinusoidal test carried out at the corresponding maximum strain level has been drawn with bold line. All the hysteretic cycles always converge to the enveloping curve obtained for the test carried out at maximum strain of $200 \%$. Moreover, the curves produced by asymmetrical tests with maximum strain of $100 \%$ follow sible to see that all re reached during the tet from the results of as mmetric loading tests that the loading or unloading path pendent on the previews straim history and state existing when the sign of the velocity changes.

Register for free at https//www.scipedia.com to download the version without the watermark
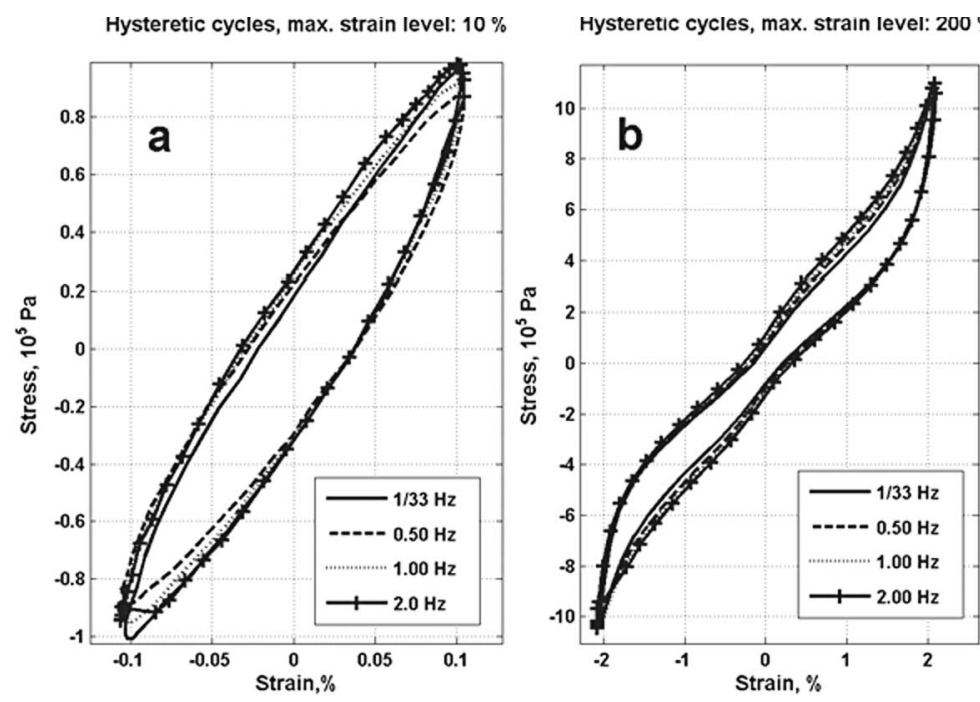

FIGURE 7 Hysteretic cycles for the following loading frequencies: 1/33, 0.5, 1.0, 2.0 Hz. (a) Maximum strain level $20 \%$. (b) Maximum strain level $200 \%$. 
Asymmetric curves and $200 \%$.

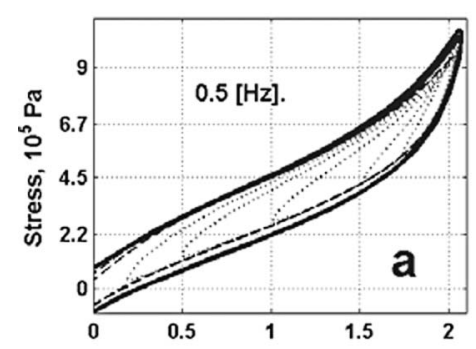

Asymmetric curves, $200 \%$ and $150 \%$

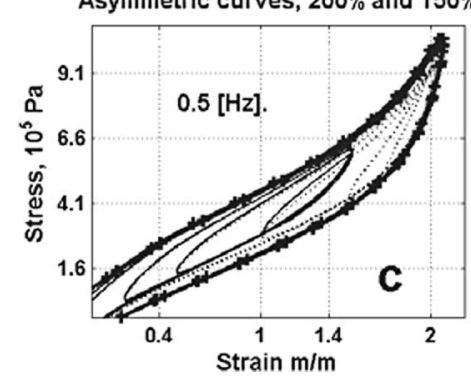

Asymmetric curves and $150 \%$.

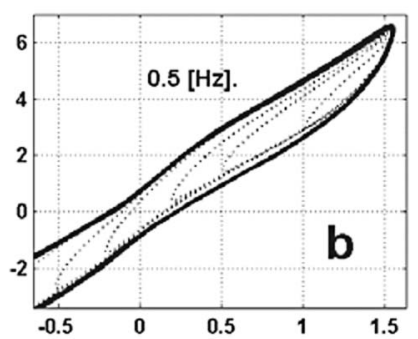

Asymmetric curves and $100 \%$.

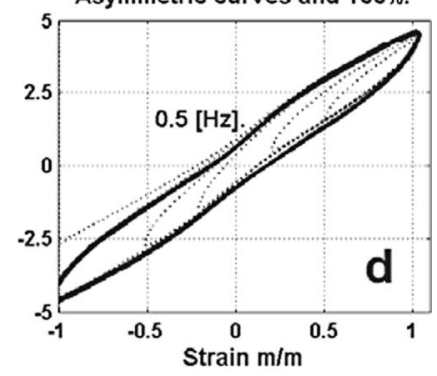

FIGURE 8 Loading cycles for different maximum strain levels. All the loading or reloading branches, $v>0$, converge to the enveloping curve of the strain-stress curves.

2.4.2 Rubber Behavior to Arbitrary Loading Paths. The response of the test specimens subjected to arbitrary loading paths shows the followings characteristics:

- The imposed sequence of ramps induces in the rubber an initial shear strain of $200 \%$ following the upper enveloping curve. After that, several asymmetric cycles are imposed at different strain levels. All these cycles are attached to the lower branch of the enveloping curve of Fig. 2c. In all cases, energy dissipation is obtained for the secondary cycles. This results allow to affirm that inelastic hysteretic behavior occurs for any strain level, confirming that the instantaneous shear modulus changes from point to point in the strain-stress space.

- In the case when the sequence of displacements obtained from a SDF system is applied, the rubber dissipates energy according with the previously described behavior. For low strain levels $(<50 \%)$, the material shows the stiffest behavior. For medium strain levels (50-150\%), the average elastic modulus decreases with respect to the low strain level, but the area of the hysteretic cycles increases. For high strain levels $(>150 \%)$, axial hardening becomes noticeable, contributing to increase stiffness again, as can be seen in Fig. 2b. This behavior allows to justify that the material is a good candidate for developing energy dissipating devices. For seismic applications in base isolation and energy dissipating devices, it is desirable to have a stiff response in low strain levels, aiming to limit lateral movements of the structures during small loadings. If high strain levels are induced during strong earthquakes, it would be desirable to stiffen the structure to avoid second order effects or impacts with adjacent structures. In the medium strain ranges, high energy dissipation is required [Kelly, 1988; Soong and Dargush, 1997]. 


\section{Proposed Numerical Model for the Strain-Stress Relationship of the Rubber}

The proposed approach for describing the strain-stress relationship of the rubber subjected to imposed displacements is based on parametric curves, which fit to the experimental data. This numerical model does not require defining constitutive laws for the material or its components in a general stress state, what is generally complicated and computer time consuming [Hwang and Ku, 1997; Salomon et al., 1999]. A simple model for high damping rubbers is described in this section to be incorporated in an efficient software package for the analysis of passively controlled structures [Valles et al., 1996].

The traditional way to treat rubber and other visco elastic materials employed in energy dissipating devices used in earthquake engineering has assumed an equivalent viscous damping added to the structure [AASHTO, 1991; Soong and Dargush, 1997]. The development of special structural elements with dissipating characteristics similar to those given by the rubber usually is a complex problem due to the necessity of incorporating frequency dependency, variable stiffness or axial hardening in the model. However, if a relatively simple mathematical model is available for rubber-like materials, the traditional design procedures, based on equivalent added damping, can be improved and it is possible to apply more sophisticated techniques such as Fast Nonlinear Analysis (FNA), which allows simulating the structural response with material nolinearities concentrated in specific dissipating devices [Wilson, 1998].

Based on the experimental results of Sec. 2, the following form of the shear strainstress relationship of the material is proposed:

$$
\sigma(\varepsilon, \dot{\varepsilon}, t)=\sigma_{1}(\varepsilon, t)+\sigma_{2}(\dot{\varepsilon}, t)
$$

where $\sigma(\varepsilon, \dot{\varepsilon}, t)$ is the average shear stress in the rubber specimen during a test, $\varepsilon$ is the shear strain level, $t$ is the time, $\dot{\varepsilon}$ is the strain rate, $\sigma_{l}$ is the component of the shear stress depending on the strain level, and $\sigma_{2}$ is the viscous component depending only on the strain rate. The proposed model uncouples the total stress in viscous and nonviscous components. The stress $\sigma$ is a phenomenological description depending on a set of parameters, which have to be calibrated from experimental data. This model is able to describe the average shear stress which appears in the material when it is subjected to shear strains, being this the predominant tensional state in energy dissipating devices based on rubberlike materials [Naeim and Kelly, 1999].

The proposed model consists of viscous dashpot device, simulating the component $\sigma_{2}$ of the stress, acting in parallel with a nonlinear hysteretic spring for the $\sigma_{1}$ component. In general, the purely viscous component, $\sigma_{2}$, does not require to be a linear function of the strain rate level. From the experimental results, it is possible to deduce that the function $\sigma_{l}$ should have the following characteristics:

- Axial hardening for strains over $150 \%$.

- Variable shear modulus for fitting the strain-stress curves obtained during the tests.

- The initial slope of a loading or unloading branch is only a function of the point in the strain-stress space where the velocity changes of sign, according to Fig. 8.

\subsection{Proposed Method to Estimate the Viscous Component}

An explicit form for the viscous component $\sigma_{2}$ of Eq. (3.1) is proposed herein. The dissipated energy increment, $\Delta E$, corresponding to two tests carried out at the same maximum 
displacement, $u$, but for different consecutive frequencies $\omega_{i+1}$ and $\omega_{i}$, $(i$ is the frequency index), it is given by

$$
\Delta E=A h\left\{\int\left(\sigma_{1}+\sigma_{2}^{i+1}\right) d \varepsilon-\int\left(\sigma_{1}+\sigma_{2}{ }^{i}\right) d \varepsilon\right\}=A h \int\left(\sigma_{2}^{i+1}-\sigma_{2}^{i}\right) d \varepsilon
$$

where $A$ and $h$ are the area and thickness of the test specimen and the term $\left(\sigma_{1}+\sigma_{2}\right)$ is the average shear stress according to Eq. (3.1). $\sigma_{l}^{\mathrm{i}}$ is the viscous component of the average shear stress for the cycles applied with loading frequency $\omega_{i}$. An additional working hypothesis is the following expression for the viscous component of the force:

$$
A \sigma_{2}=h c(\omega) \dot{\varepsilon}=h c(\dot{\varepsilon}) \dot{\varepsilon}
$$

To obtain a numerical estimation for the function $\sigma_{2}$, it is possible to suppose that, for a test carried out with a loading frequency $\omega_{i}$ and maximum displacement $u_{j}$, dissipation is related to velocity through the damping coefficient, $c_{i j}$. Considering only symmetric tests, Eq. (3.2) can be rewritten as

$$
\begin{gathered}
\dot{\varepsilon}\left(u_{j}, \omega_{i}\right)=\frac{u_{j}}{h} \omega_{i} \cos \left(\omega_{i} t\right) \\
\Delta E=u_{j}^{2} \omega_{i+1}^{2} \int_{0}^{T_{i+1}} c_{(i+1) j} \cos ^{2}\left(\omega_{i+1} t\right) d t-u_{j}^{2} \omega_{i}^{2} \int_{0}^{T_{i}} c_{i j} \cos ^{2}\left(\omega_{i} t\right) d t .
\end{gathered}
$$

If the values of the two loading frequencies are close, we can suppose that the coefficients $c_{i j}$ are approximately the same, i.e, $c_{(i+1), j}=c_{i j}$, and calculate them as follows:

$$
c_{i j}=\frac{\Delta E}{u_{j}^{2}\left\{\omega_{i+1}^{2} \int_{0}^{T_{i+1}} \cos ^{2}\left(\omega_{i+1} t\right) d t-\omega_{i}^{2} \int_{0}^{T_{i}} \cos ^{2}\left(\omega_{i} t\right) d t\right\}}
$$

It is possible to fit a polynomial to the values of all the coefficient $c_{i j}$ obtained from sinusoidal tests for two different consecutive frequencies and fixed displacement level. This polynomial is a function of the frequency and describes the damping coefficient of Eq. (3.5). For the case of the studied material, the bigger dispersion is obtained for values of the damping coefficient in the range $0.033-0.5 \mathrm{~Hz}$, because in this set of tests a non viscous part of the dissipated energy is involved. For higher frequency values, this effect disappears according to Eq. (3.2). If the values for the first range of the tests are omitted, it is possible to consider for this high damping rubber an average value for $C$ of $1.20 \mathrm{Ns} / \mathrm{m}$. Thus, Eq. (3.1) takes the form

$$
\sigma=\sigma_{1}+\frac{h}{A} 0.12 \dot{\varepsilon}
$$




\subsection{Proposed Model for the Non-viscous Component}

3.2.1 Axial Strain Hardening. The capacity of the model to simulate axial hardening for strain levels over $150 \%$ is given by an appropriated nonlinear elastic backbone added to the non viscous hysteretic cycles, as it can be seen in the scheme of Fig. 9 [Naeim and Kelly, 1999]. The proposed backbone is defined numerically by means of a polynomial, whose coefficients are fitted to experimental data. Details about the fitting method are given by Mata et al. [2005]. The following hardening function is obtained:

$$
\begin{gathered}
\sigma_{1 \_ \text {hardening }}=\operatorname{sgn}(\varepsilon) A_{o}(\langle|\varepsilon|-1.5\rangle)^{1.5} \quad\left(A_{o}=5.0\right) \\
\operatorname{sgn}(\varepsilon)=\frac{\varepsilon}{|\varepsilon|} \\
\langle\varepsilon\rangle=\frac{1}{2}(\varepsilon+a b s(\varepsilon))
\end{gathered}
$$

The sign and absolute value functions in Eq. (3.7) allow to develop axial hardening in both quadrants of the strain-stress plane.

3.2.2 Non Viscous Hysteretic Cycles. The proposed model for the non viscous part of the hysteretic cycles modifies the plasticity model proposed by Wilson, [1998]. The restoring force model employed in this reference is obtained by solving the following system of differential equations by means of the finite difference method

$$
\begin{aligned}
& \sigma=k_{y} \varepsilon+\left(k_{e}-k_{y}\right) e \\
& \text { if } \quad \dot{\varepsilon} e \geq 0 \quad \rightarrow \quad \dot{e}=\left(1-\left|\frac{e}{\varepsilon_{y}}\right|^{n}\right) \dot{\varepsilon} \\
& \text { else } \quad \rightarrow \quad \dot{e}=\dot{\varepsilon}
\end{aligned}
$$

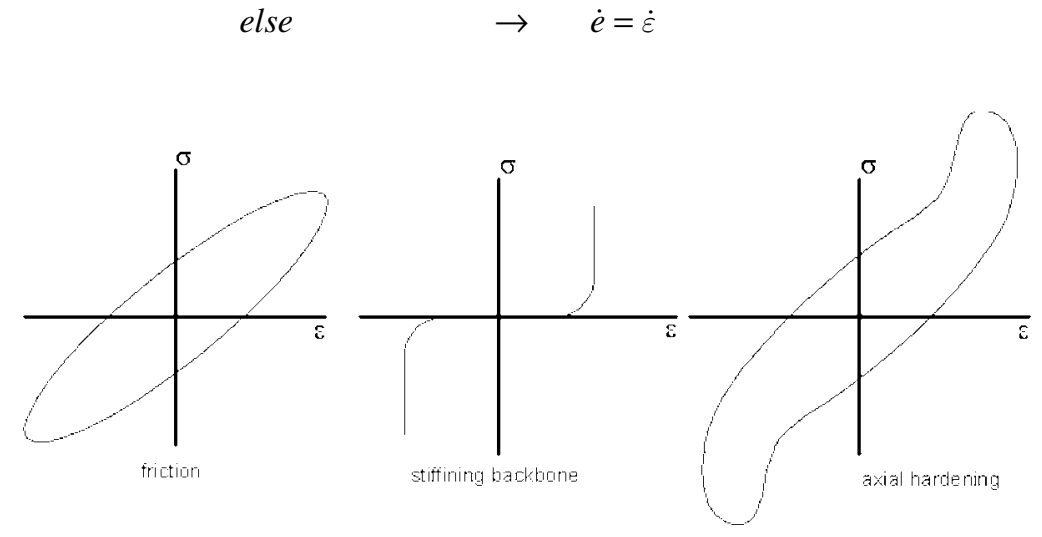

FIGURE 9 Schematic representation of the method employed for including axial hardening into the model. An non linear elastic backbone is added to a hysteretic cycle. 
where $\sigma$ is the stress, $K_{y}$ is the post yielding stiffness, $K_{e}$ the elastic stiffness, $\varepsilon$ the real strain of the system, $\varepsilon_{y}$ is the yielding strain of the material, and $e$ represents an internal variable of plastic (hysteretic) strain, which takes a values in the range $\left[-\varepsilon_{y}, \varepsilon_{y}\right]$. The parameter $n$ in the associated flow rule of Eq. (3.8) describes the degree of smoothness exhibited by the transition zone between the pre- and the post-yielding branches of the hysteretic cycle [see SAP, 2004]. A low value of $n$ implies a smoother transition and, as $n$ increases, the model approximates a bilinear curve, as it can be seen in Fig. 10.

The proposed model for the component $\sigma_{I}$ solves the system of Eqs. (3.8) taking into account that the parameters of the model, $K_{e}, K_{y}, d_{y}$, and $n$, are function of the point in the strain-stress space where the last change of sign of the strain rate, $\dot{\varepsilon}$, has occurred, that is, when the system goes from loading to unloading or vice versa. Therefore, the proposed algorithm updates the parameters of the model for each change of sign of the strain rate. If there is no change in the sign of $\dot{\varepsilon}$, the parameters of the model are maintained constants. Thus, the system of equations to solve is

$$
\begin{gathered}
\sigma_{1}=k_{y}\left(\varepsilon^{c v}, \sigma^{c v}\right) \varepsilon+\left(k_{e}\left(\varepsilon^{c v}, \sigma^{c v}\right)-k_{y}\left(\varepsilon^{c v}, \sigma^{c v}\right)\right) e \\
\text { if } \dot{\varepsilon} e \geq 0 \quad \dot{e}=\left(1-\left|\frac{e}{\varepsilon_{y}\left(\varepsilon^{c v}, \sigma^{c v}\right)}\right|^{n\left(\varepsilon^{c v}, \sigma^{c v}\right)}\right) \dot{\varepsilon} . \\
\text { else } \rightarrow \quad \dot{e}=\dot{\varepsilon} \\
k_{e}=\phi_{1}\left(\varepsilon^{c v}, \sigma^{c v}\right), k_{y}=\phi_{2}\left(\varepsilon^{c v}, \sigma^{c v}\right), d_{y}=\phi_{3}\left(\varepsilon^{c v}, \sigma^{c v}\right), n=\phi_{4}\left(\varepsilon^{c v}, \sigma^{c v}\right)
\end{gathered}
$$

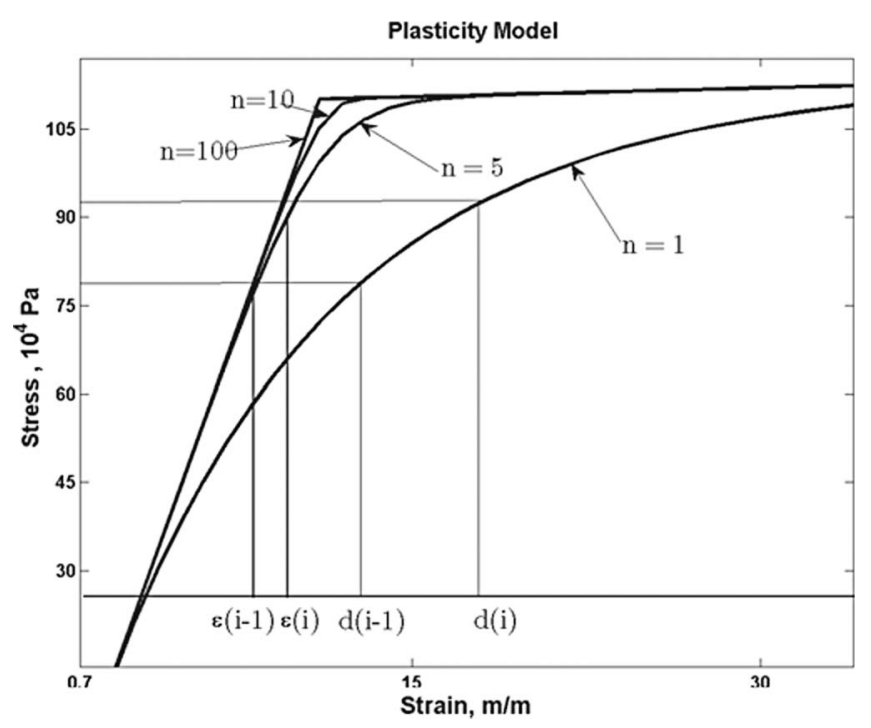

FIGURE 10 Plasticity model. Smooth transition between pre- and post-yielding stiffness. As the factor $\mathrm{n}$ is increased, the behaviour approximates to a bilinear one. 
where $\varepsilon_{v}$ is the yielding strain and $\left(\varepsilon^{c v}, \sigma^{\mathrm{cv}}\right)$ are the strain and stress levels existing in the point when the sign of $\dot{\varepsilon}$ changes. In Eqs. (3.9) and (3.10), it has been emphasized the dependence between the parameters of the model and the point where $\dot{\varepsilon}$ changes the sign. Explicit forms for the functions $\phi_{1}, \phi_{2}, \phi_{3}$, and $\phi_{4}$ have to be determined from experimental data.

A scheme of the updating procedure is shown in Fig. 11. The flow chart of the algorithm that integrates the system of Eqs. (3.9) and (3.10) is shown in Fig. 12. The algorithm starts by assigning initial values to the parameters of the model. For each strain level $\varepsilon_{i}$ the algorithm verifies if the strain rate, $\dot{\varepsilon}$, changes of sign. If this is the case an updating procedure for the parameters $\phi_{1}, \phi_{2}, \phi_{3}$, and $\phi_{4}$ is carried out. On the contrary, the parameters are maintained. The plastic strain and stress are then estimated.

3.2.3 Determination of Functions $\phi_{1}, \phi_{2}, \phi_{3}, \phi_{4}$. The function $\phi_{1}$ defines the pre-yielding stiffness of the strain-stress curve. An additional simplification is made in this article consist in supposing that $\phi_{1}$ is a function only of $\varepsilon_{c v}$, which is the strain level existing when $\dot{\varepsilon}$ changes the sign. Applying the methods proposed by Mata et al. [2004], it is possible to develop a polynomial function describing the instantaneous stiffness after the change in the sign of $\dot{\varepsilon}$. The values of the coefficients of the polynomial for the case of the studied material are given by

$$
\begin{aligned}
\phi_{1}\left(\varepsilon_{c v}\right)= & -0.14+0.95 \varepsilon_{c v}-2.45 \varepsilon_{c v}^{2}+3.19 \varepsilon_{c v}^{3}-2.25 \varepsilon_{c v}^{4} \\
& +0.89 \varepsilon_{c v}^{5}-0.20 \varepsilon_{c v}^{6}+0.04 \varepsilon_{c v}^{7} .
\end{aligned}
$$

The precision of the obtained function is dependent on the quality of the experimental data.

The post-yielding stiffness function, $\phi_{2}$, is maintained constant and equal to the value of the slope of the tangent to the enveloping curve of the test carried out for a strain level of $200 \%$, due to the fact that the tests show that all the loading or unloading branches finally converges to the enveloping curve. Therefore, $\phi_{2}$ is calculated as follows

$$
\phi_{2}\left(\varepsilon_{c v}, \sigma_{c v}\right) \approx K_{y}=3.30
$$

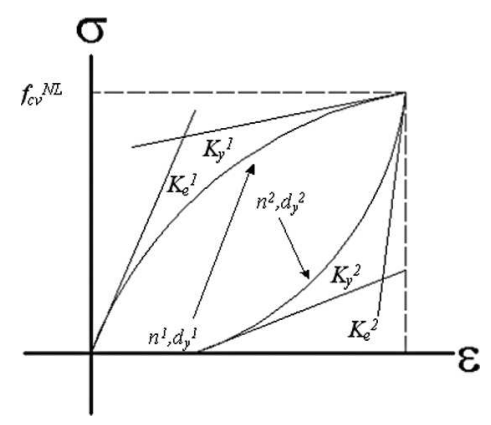

FIGURE 11 Proposed model. The initial curve is obtained integrating the plastic model for the initial values of the parameters $K e^{l}, K y^{l}, n^{l}$, and $d y^{l}$. After the loading-unloading transition have occurred, the parameters of the model are updated to $K e^{2}, K y^{2}, n^{2}$, and $d y^{2}$. 


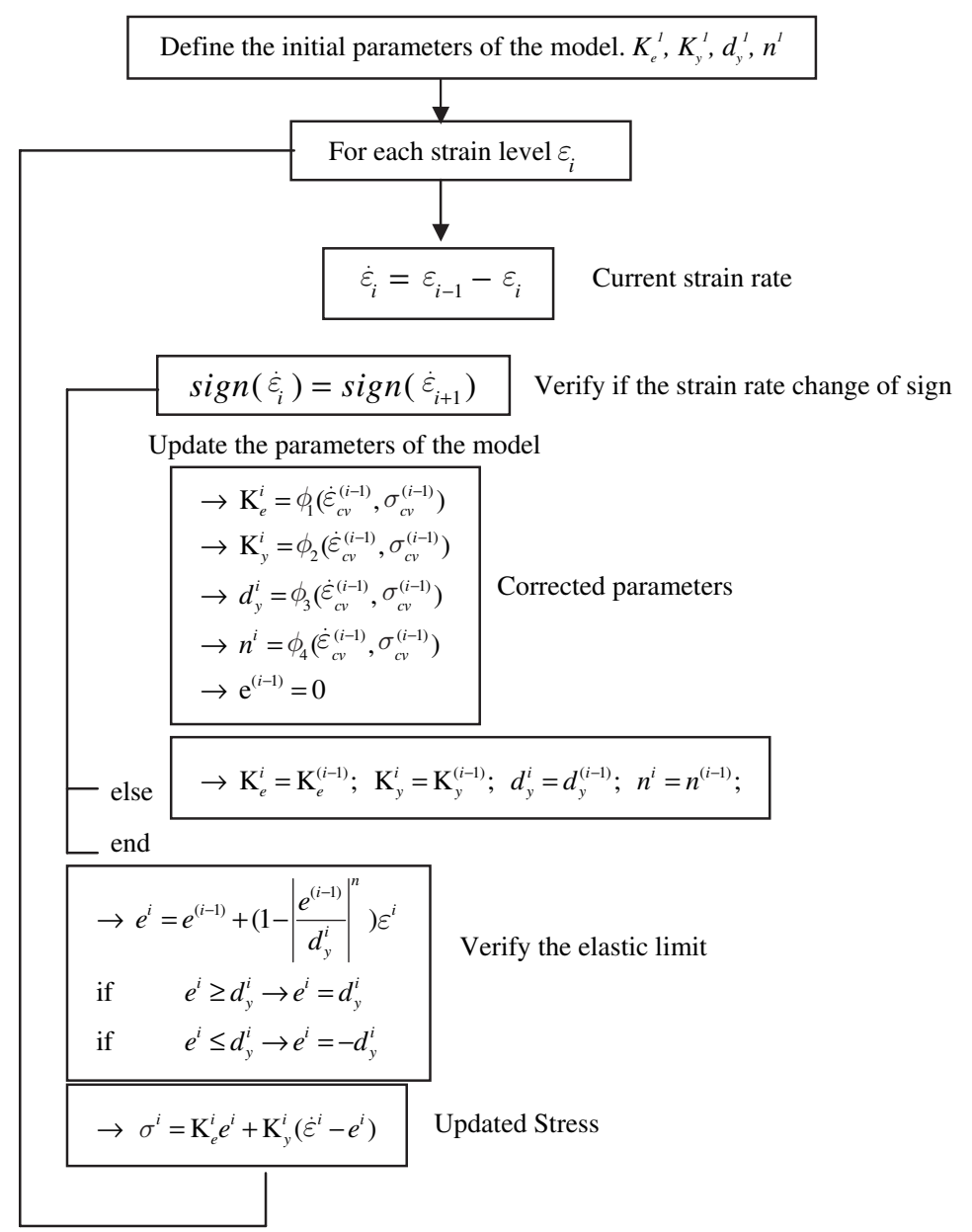

FIGURE 12 Flow chart for the integration algorithm for the Eqs. (3.9) and (3.10).

The yield strain function $\phi_{3}$ is obtained intersecting the line with slope $\phi_{1}$ and the enveloping line with slope $\phi_{2}$. The yield strain is measured from the point where $\dot{\varepsilon}$ changes of sign to the intersecting point. The analytical expression for $\phi_{3}$ is

$$
\phi_{3}\left(\varepsilon_{c v}, \sigma_{c v}\right) \approx \phi_{3}\left(\sigma_{c v}\right)=\frac{\left|\sigma_{c v}\right|-\left|\sigma_{0}-\sigma_{c v}\right|}{\left(\phi_{2}-\phi_{1}\right)}
$$

where $\sigma_{c v}$ is the stress level existing when $\dot{\varepsilon}$ changes the sign and $\sigma_{0}$ is a parameter calculated evaluating the line with slope $\phi_{2}$ at zero strain level.

The function $\phi_{4}$ has to take values within the range $[1, \infty]$. Simulations considering increasing values for $\phi_{4}$ where carried out to approximate each curve obtained from symmetric tests. For each value of $\phi_{4}$, the differences between the predicted values for the stresses and the experimental data were computed. The square root of the absolute value of the sum of those differences was chosen as function to be minimized. The obtained results allow to conclude that the best value for $\phi_{4}$ was

$$
\phi_{4}\left(\varepsilon_{c v}, \sigma_{c v}\right) \approx \phi_{4}=1.0 \text {. }
$$




\subsection{Proposed Model}

Having determined the functions $\phi_{1}, \phi_{2}, \phi_{3}$, and $\phi_{4}$, the corresponding form for the model of Eq. (3.6) is now given by

$$
\left.\sigma_{1}=\phi_{2} d\left(\varepsilon^{c v}, \sigma_{N L_{c v}}\right)+\left(\phi_{1}\left(\varepsilon^{c v}\right)-\phi_{2}\right) e+\operatorname{sgn}(\varepsilon) 5.0(|\varepsilon|-1.5\rangle\right)^{1.5}+0.12 \dot{\varepsilon}
$$

This model is able to simulate: energy dissipation for cuasi static loading, variable shear modulus, axial hardening for strains over $150 \%$, energy dissipation due to viscous mechanisms, and history independent behavior. Ten parameters are needed to characterize the proposed model, which can be considered a large number comparing with the number of parameters required by other models in literature [see, e.g., Wen, 1976; Soon and Dargush, 1997]. But, on other hand, relatively simple procedures are required for their calibration from experimental data and this fact and the versatility showed by the model compensate the higher number of variables.

\section{Validation of the Proposed Model}

The response predicted by the proposed model is compared with experimental data for symmetric and arbitrary loading cases to validate its capacity to simulate the rubber behavior. Additionally, the responses obtained from Kelvin and Plasticity models are compared with the model response and the experimental data.

\subsection{General Behavior of the Model}

For highlighting the ability of the proposed model in simulating the general behavior of rubbers, fictitious values for the parameters $\phi_{1}, \phi_{2}, \phi_{3}$, and $\phi_{4}$ are employed when subjecting the model to a sinusoidal record of imposed displacements with an increasing strain level up to $200 \%$ and a loading frequency of $0.5 \mathrm{~Hz}$. A damping coefficient value of $1.20 \mathrm{Ns} / \mathrm{m}$ determined from experimental data and other two other fictitious values of $0.50 \mathrm{Ns} / \mathrm{m}$ and $3.0 \mathrm{Ns} / \mathrm{m}$, were employed to compare their influence in the response. It is possible to see from Fig. 13b that energy dissipation is increased when the viscous damping coefficient is increased. The model shows variable shear modulus and it is possible to distinguish three zones:

- Low strain level, $<50 \%$, which corresponds to the highest values of the shear modulus.

- Medium strain level, 50-150\%, which corresponds to moderate values of the shear modulus.

- Large strain values, $>150 \%$, for which instantaneous stiffness is increased again and axial hardening becomes apparent, allowing to simulate the configuration changes of the test specimens during the experiments.

The hysteretic cycles show an elliptical shape for small strains, but when they are increased, the cycles approximate to a rectangle.

A second set of simulations was carried out to evaluate the ability of the model to dissipate energy for the same record of imposed displacements of Fig. 13a, but different loading frequencies. Results for a damping coefficient of $1.20 \mathrm{Ns} / \mathrm{m}$ are shown in Fig. 14a. In this case, the loading frequencies were $1 / 33,1.00$, and $2.00 \mathrm{~Hz}$. It is possible to see the non viscous part of the dissipated energy for loading frequency of $1 / 33 \mathrm{~Hz}$. The bigger the 

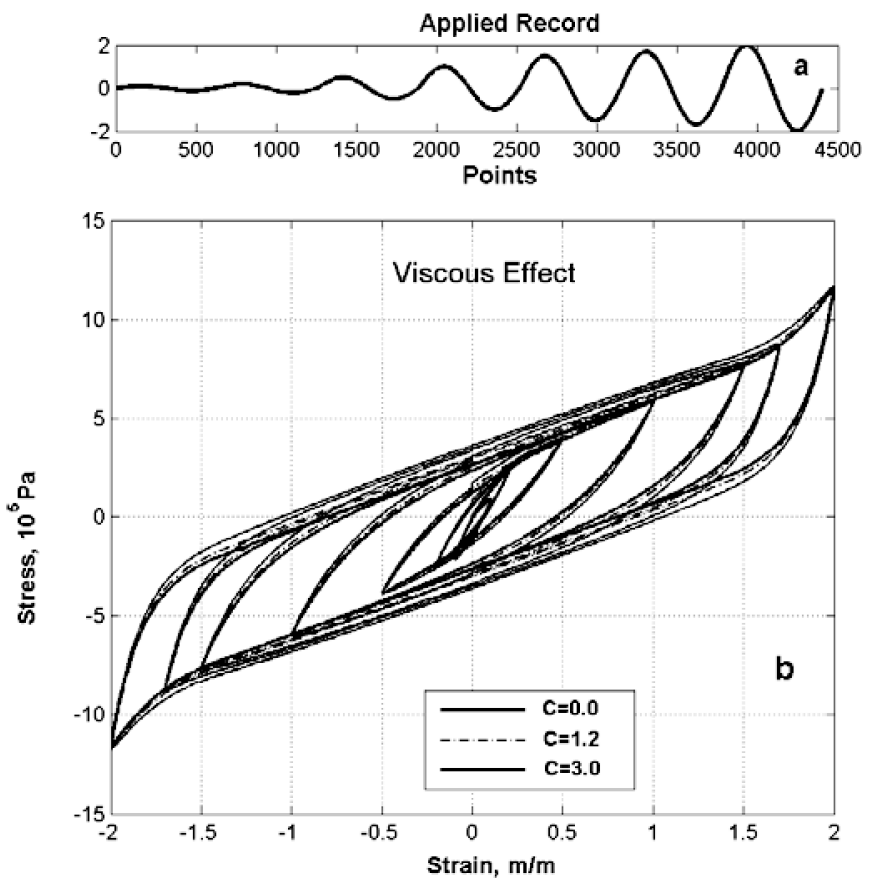

FIGURE 13 (a) Applied record of imposed displacements. (b) Hysteretic cycles obtained for three values of the damping coefficient.

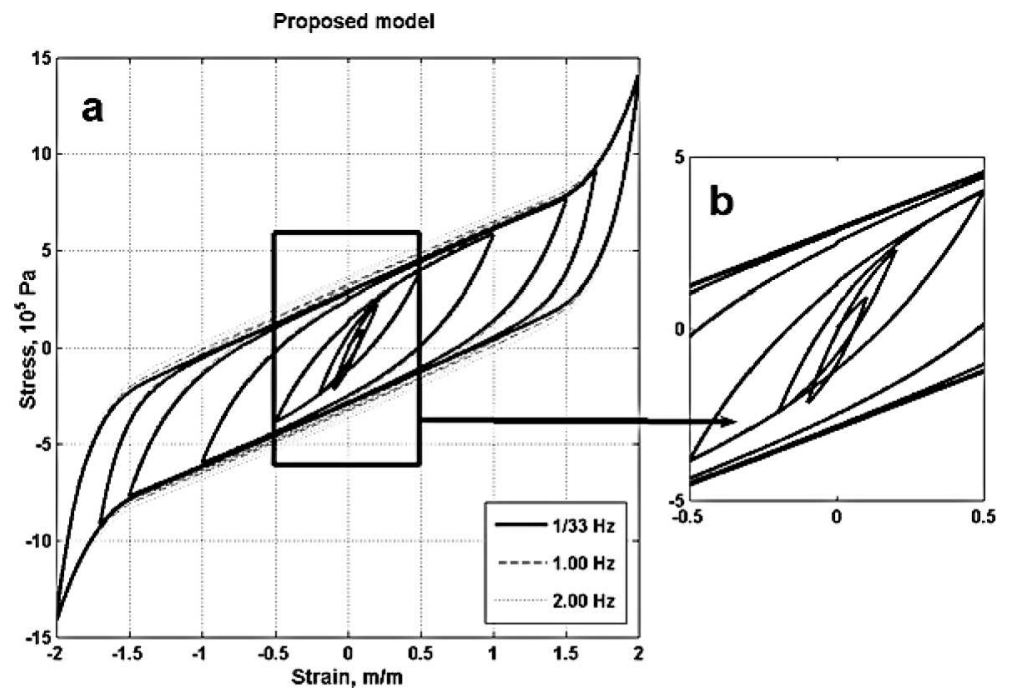

FIGURE 14 Hysteretic cycles. (a) Results for different loading frequencies. (b) Detail of the zone corresponding to low strain level.

loading frequency the bigger the dissipated energy. In Fig. 14b, there is a detail of the low strain zone where it is possible to appreciate the shape of the hysteretic cycles initially elliptic but, when the strain level is increased, the ellipse is enlarged along its major axis approximating its shape to a rectangle. 


\subsection{Comparison with Experimental Data Subjected to Symmetric Loading Tests}

The response of the model calibrated from experimental data was compared with results from tests for a loading frequency of $0.5 \mathrm{~Hz}$. Figure 15 shows the predicted and experimental responses. A good agreement is obtained for all strain levels, but the fitting is better for strain levels over $50 \%$. The results shown in this figure allow to confirm the capacity of the model to reproduce the rubber behavior including variable shear modulus, axial hardening and the global change in the shape of the hysteretic cycle, going from approximately elliptic to rectangular.

\subsection{Comparison with Experimental Data Subjected to Arbitrary Loading Tests}

Figures $16 \mathrm{a}, 16 \mathrm{~b}, 17 \mathrm{a}$, and $17 \mathrm{~b}$ show the numerical results for the simulation of the rubber response for arbitrary loading records. For the case of an arbitrary sequence of ramps, it is possible to see a general good agreement between the predicted stress and experimental data. The differences can be explained by the fact that the initial stiffness is taken as function only of the strain level existing when the strain rate change of sign and, therefore, it does not take into account the stress level. From Fig. 16a it is possible to appreciate that energy dissipation occurs during the cycles induced by the ramps; however, the agreement between model and experimental data shows more significant differences for medium strain levels. On the other hand, the model is able to reproduce the enveloping cycle with a remarkable precision. The general behavior of the rubber is well fitted by the model including variable stiffness, energy dissipation in asymmetric loading cycles, and axial hardening.

For the case of the displacement record obtained from the seismic record, the comparison between numerical and experimental data is shown in Fig. 17. In Fig. 17a, it is possible to see a good agreement between the hysteretic cycles obtained through numerical simulation and experimental data. The model is able to reproduce variable stiffness and axial hardening. The good agreement is confirmed by Fig. 17b, where the predicted stress time history fits the experimental values with an error of $5 \%$.

Finally, it is possible to say that the proposed numerical model is able to describe the rubber behavior for an arbitrary loading case with remarkable precision. The differences between numerical and experimental data can be explained by the fact that the initial stiffness function only depends on the strain level.

\subsection{Comparison with Other Models}

The numerical response of the model is now compared with those obtained by means of Kelvin's model and the plasticity model. Kelvin's model is frequently used in case of elastomers with a high content in visco elastic rubber, because the most of the dissipated energy is due to viscous mechanisms [Sommer, 1989]. When the content of black hums in the rubber is increased, the non viscous hysteresis became predominant in the response [Salomon et al., 1999; Ponce León et al., 1997]. In this case, the plasticity model is able to simulate the mechanical response more realistically than Kelvin's. The two models are probably not able to simulate realistically the rubber behavior in a general case, because the major part of the high damping rubber dissipates energy by mean of a set of phenomena including viscous, displacement dependent and frictional mechanisms among others.

Kevin's model is based on a linear spring acting in parallel with a linear dashpot. Therefore, the elastic modulus, $E$, and the viscous damping coefficient, $C$, of the material are maintained constants for any strain level [Ahmadi and Muhr, 1997]. However, the 

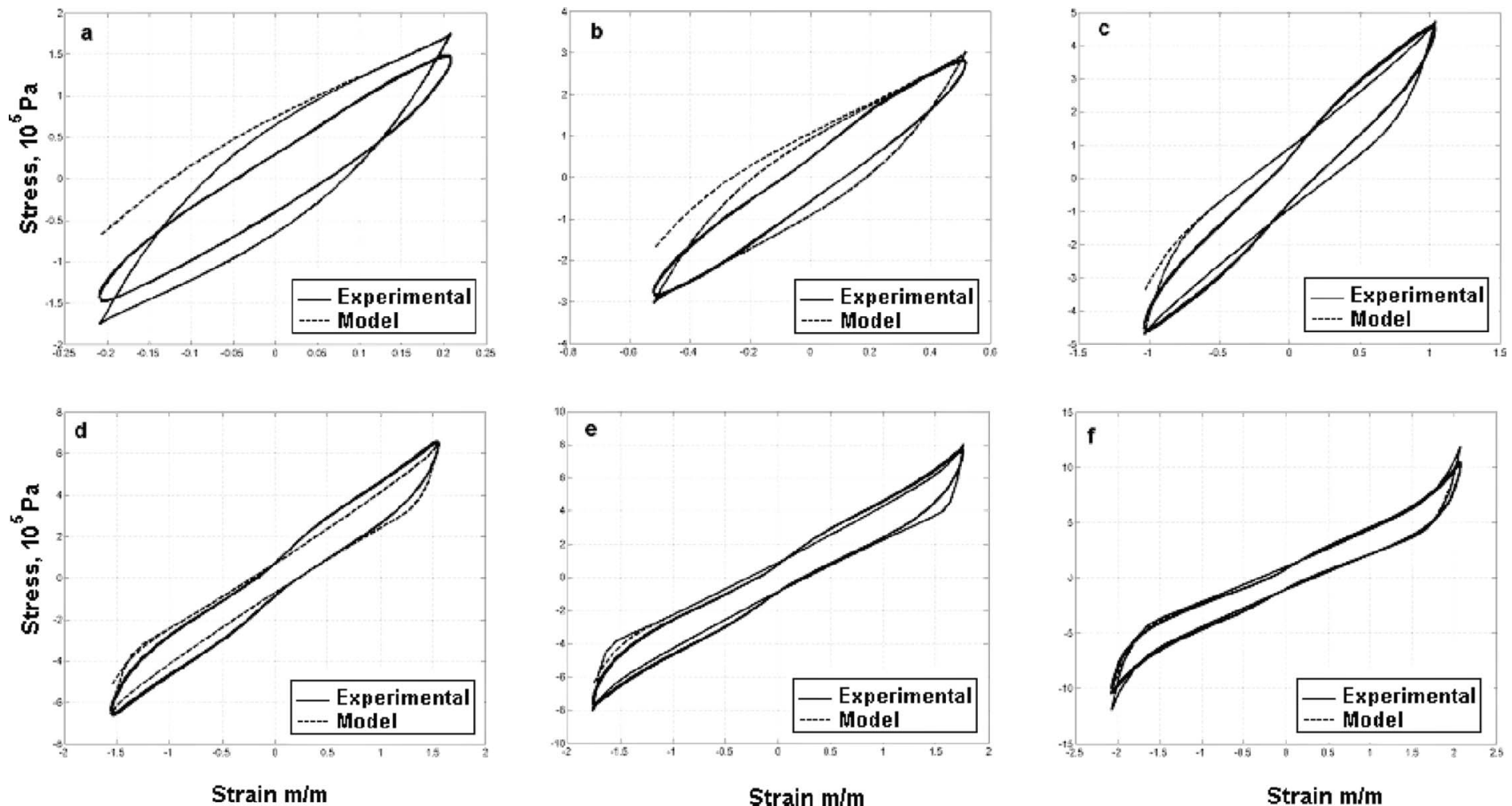

FIGURE 15 Comparison between the predicted response and experimental data for symmetric tests, for $0.5 \mathrm{~Hz}$. Maximum strain levels: (a) 20\%. (b) $50 \%$. (c) $100 \%$. (d) $150 \%$. (e) $170 \%$. (f) $200 \%$. 


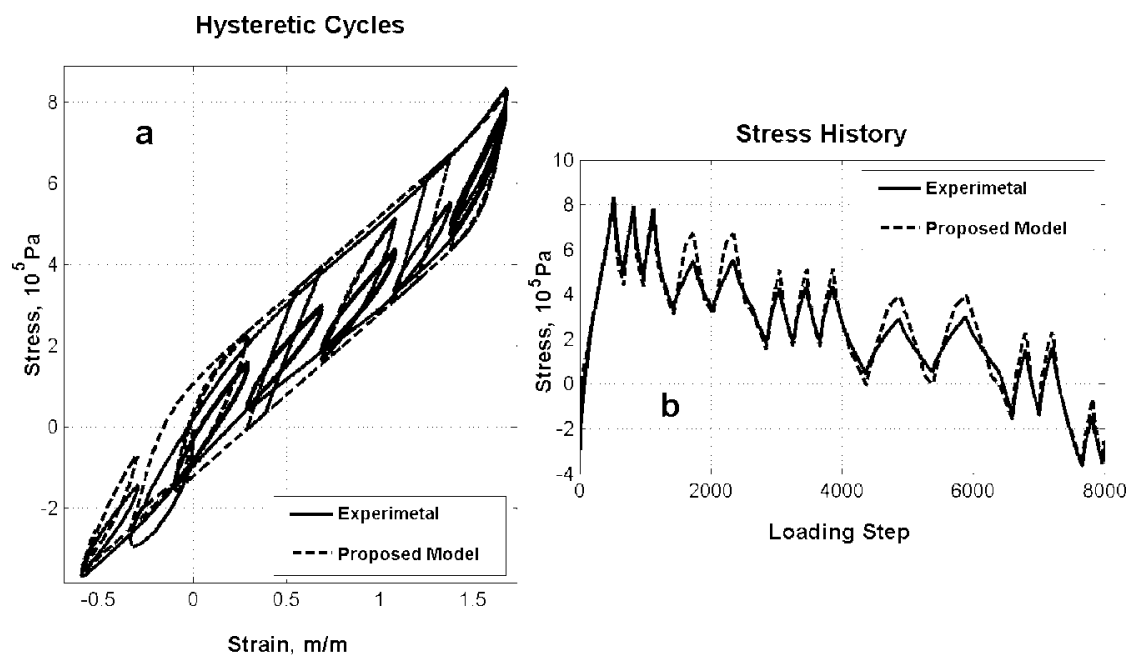

FIGURE 16 (a) Comparison between numerical simulation and experimental data for the sequence of ramps. (b) Stress time history.

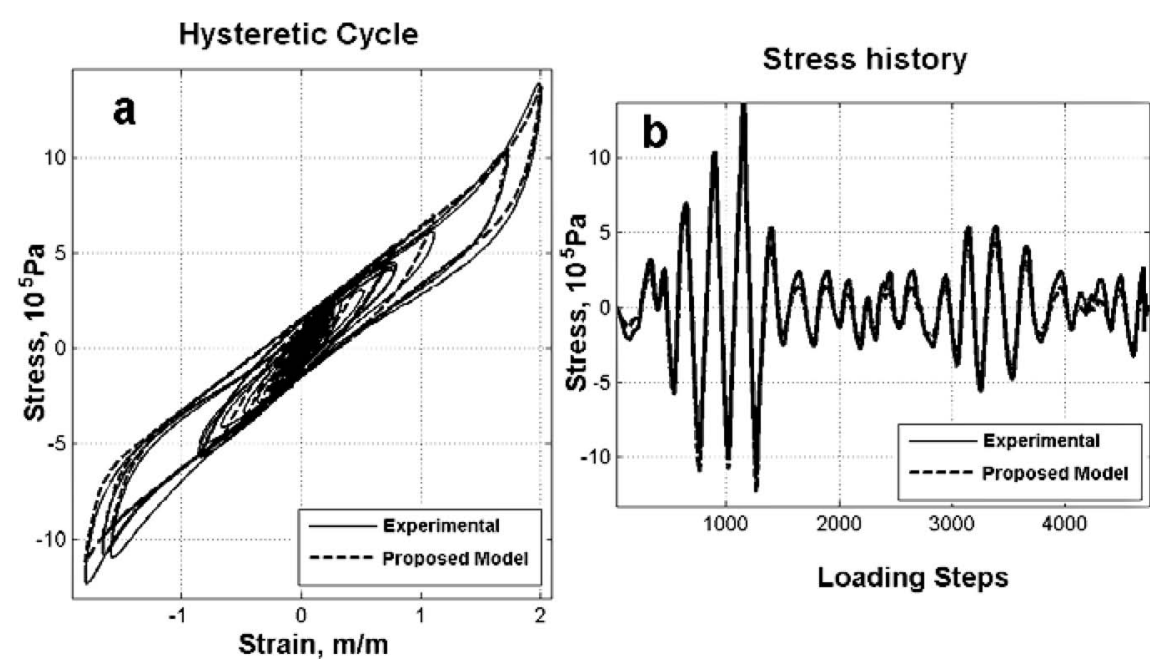

FIGURE 17 (a) Comparison between numerical simulation and experimental data for the seismic case. (b) Stress time history.

rubber frequently shows a higher stiffness for low strain levels leading to a softer behavior for medium strain levels.

Thus, if the parameters of Kelvin's model are calibrated for the simulation of the rubber behavior, two set of parameters are required. The first one is valid only for describing the rubber behavior in the medium strain range and the second one in the low strain level. Axial hardening cannot be simulated by using the this model. Good fittings in the low strain range will produce poor results in the medium range and vice versa [Mata and Boroschek, 2001]. It is possible to see in Fig. 18b the comparison between the responses obtained with the proposed model for an arbitrary record and the experimental data. The employed 

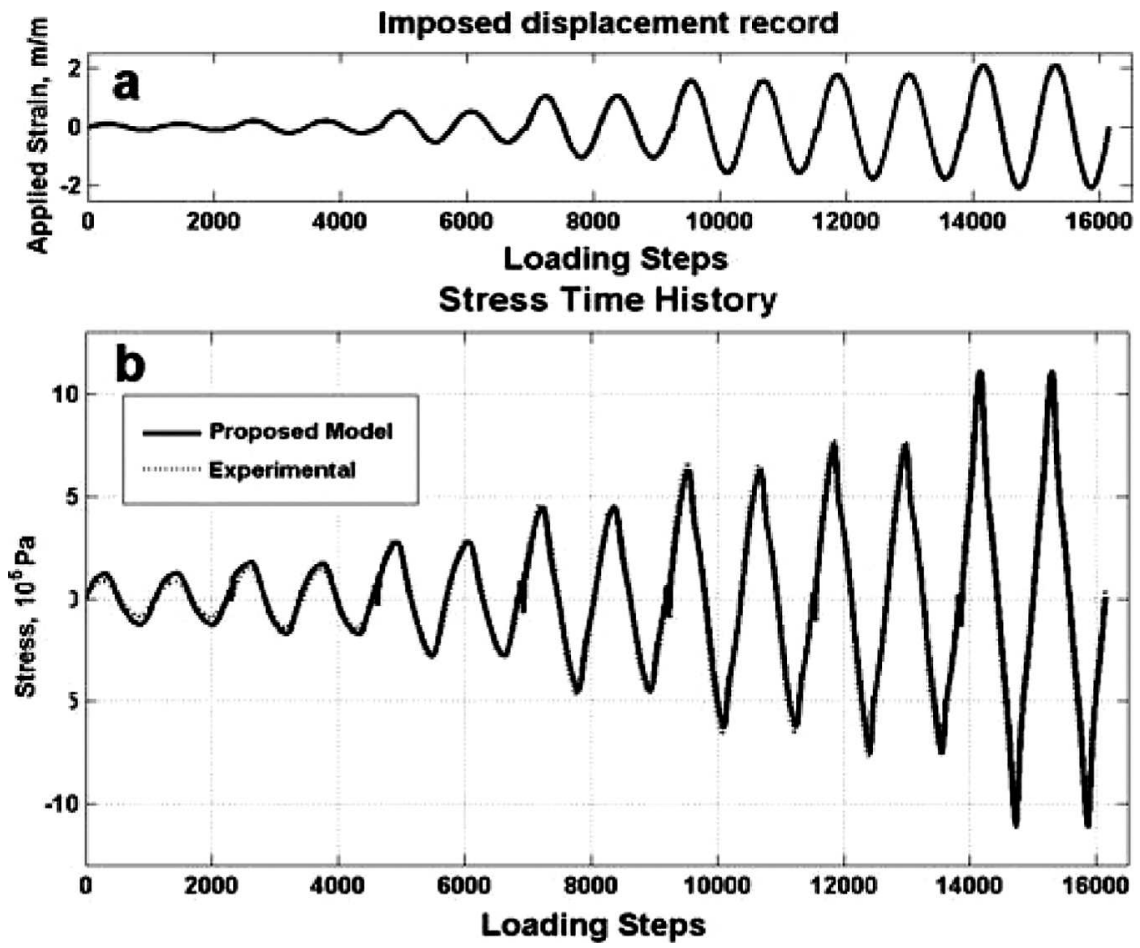

FIGURE 18 (a) Imposed displacement record. (b) Comparison between experimental data and the proposed model response.

record is shown in Fig. 18a. It is possible to see the good agreement between predicted and experimental data, (error $=\mid$ predicted value - experimental value $|/|$ exp. value $\mid<2 \%$ ).

The same record was used to study the response of Kelvin's model. Figure 19a shows the response of Kelvin's model calibrated for the set of parameters that produce a good fitting in the medium strain range. It is possible to appreciate a bad agreement for stresses in the strain range: 70-200\%. In Fig. 19b, the experimental data is compared with the response of Kelvin's model calibrated in the low strain level. It is possible to see the bad agreement of Kelvin's model for strain levels over 50\%. It is possible to conclude from Figs. 18 and 19 that the performance of the proposed model is clearly better than Kelvin's model in the case of the employed record.

The same test was applied to the case of the bilinear plasticity model. This model is defined by means of three parameters: pre-yielding elastic modulus $K_{e}$, yielding strain $f_{y}$, and post-yielding modulus $K_{y}$. The model is able to dissipate energy by non viscous mechanisms. Two sets of parameters were calibrated to fit the experimental response in the low and medium strain ranges.

Figure 20a shows the comparison between experimental data, the response from the proposed model and the plasticity model calibrated for medium strain levels. It is concluded that a better fitting is obtained for the proposed model, whereas the plasticity model shows remarkable differences (error $>8 \%$ ) for the strain range: $<70 \%$ and $>150 \%$. Figure 20b shows the same comparisons as Fig. 20a but the plasticity model was calibrated for the low strain level. In this case, the differences between the plasticity model and the experimental data can reach a maximum error of $27 \%$ for stresses in the strain range: $50-200 \%$. 
Kelvin model calibrated for large strain level

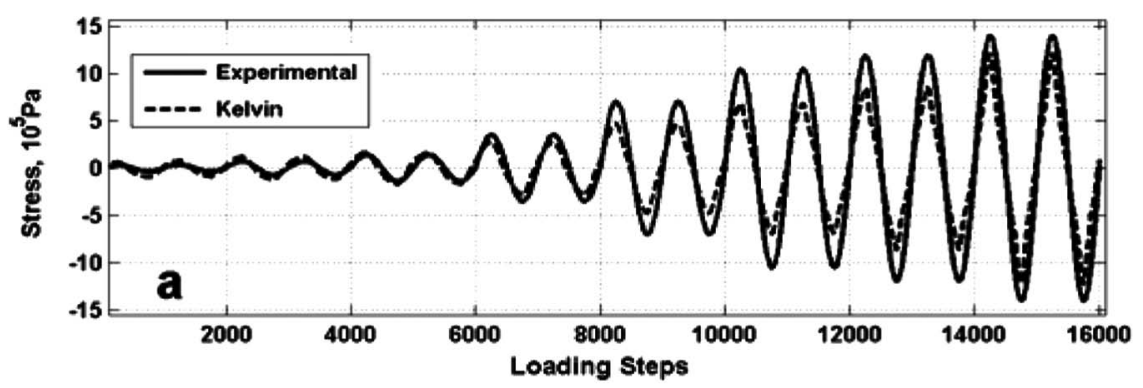

Kelvin model calibrated for small strain level

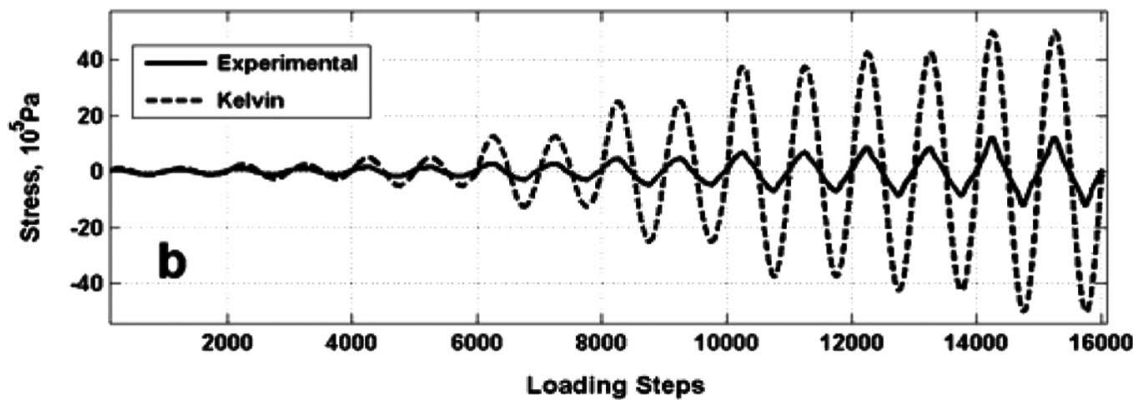

FIGURE 19 Comparison between the results predicted by the Kelvin model and the experimental data. (a) Kelvin model employing the first set of parameters: $K=3.30 .10^{5} \mathrm{~Pa}$; $C=2.20 \mathrm{~N} / \mathrm{s}$. (b) Second set of parameters $K=18.00 .10^{5} \mathrm{~Pa} ; C=3.50 \mathrm{~N} / \mathrm{s}$.
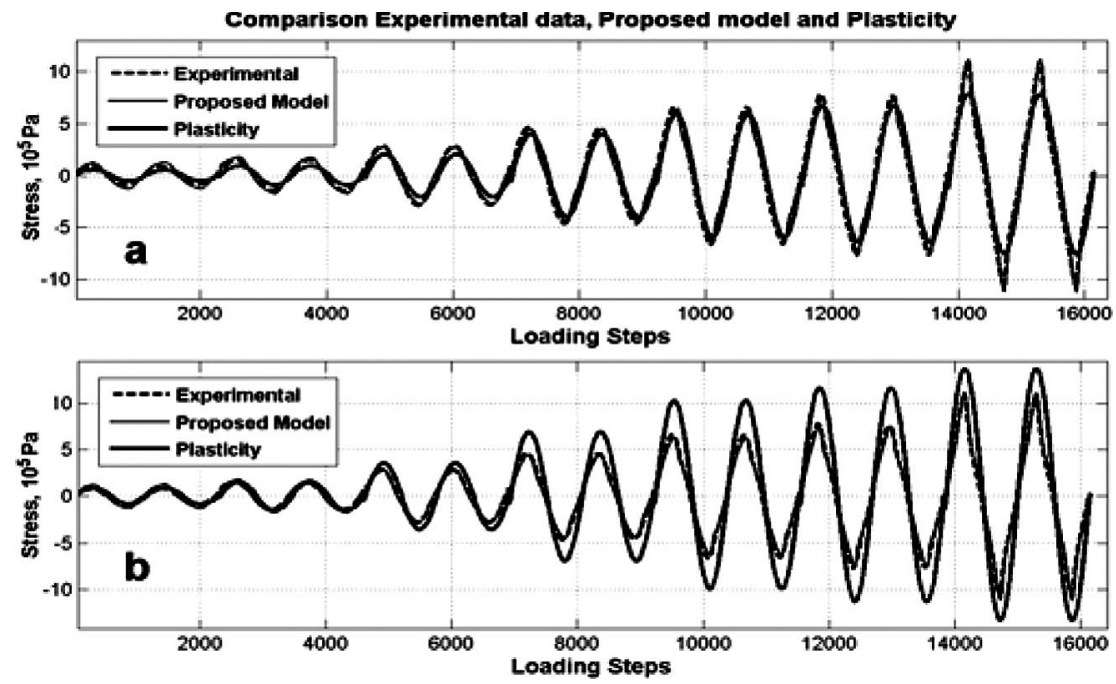

FIGURE 20 Comparison between experimental data and the responses obtained from the plasticity and the proposed models. (a) Plasticity calibrated for medium strain levels. Parameters: $K e=7.00 .10^{5} \mathrm{~Pa} ; K y=3.70 .10^{5} \mathrm{~Pa} ; f y=0.042 \mathrm{~cm} / \mathrm{cm}$. (b) Plasticity calibrated for low strain levels. Parameters: $K e=12.00 .10^{5} \mathrm{~Pa} ; K y=6.50 .10^{5} \mathrm{~Pa} ; f y=0.031 \mathrm{~cm} / \mathrm{cm}$. 


\section{Conclusions}

The mechanical characteristics of a high damping rubber to be used to develop passive control devices were studied in this work. A set of tests on rubber specimens was carried out to know the mechanical response of the rubber when it is subjected to symmetrical, asymmetrical and arbitrary records of imposed displacements. The dependency of the response with frequency and strain level was studied. The main dissipative mechanisms were identified and classified in non viscous and viscous, according to the dependency of dissipation on displacement or velocity. A method to estimate a viscous damping coefficient characterizing the velocity dependent part of the response is proposed. The displacement dependent part of the dissipation was studied, concluding that the response of material do not depend on the previous strain history. Among the more remarkable characteristics of the non viscous hysteresis are the following: strongly variable shear modulus, axial hardening for strain levels over $150 \%$, and appreciable changes in shape of the hysteretic cycles when the strain level is increased. Additionally, a new model for the rubber behavior was proposed as an extension of the plasticity model proposed in reference [Wilson, 1998]. The new model is developed adding the effects of three components: a nonlinear viscous dashpot acting in parallel with a nonlinear elastic backbone and a hysteretic spring.

All the parameters of the proposed model were calibrated from experimental data. The hysteretic spring response is obtained by solving the system of differential equations describing the relationship between the average stress and the average strain by using the finite differences method. The proposed model is validated with the data obtained from symmetrical and arbitrary tests. Moreover, the response is compared with those obtained from Kelvin's model and plasticity model. In both cases, the proposed model reproduces the rubber response with a higher precision. Several limitations for simulating rubber behavior associated with Kelvin's and the plasticity models are related with the fact that they are defined by a set of constant parameters. In the case of the proposed model, most of the parameters are defined by functions, what allows simulating more complex material behaviors.

\section{Acknowledgments}

This work has been partially supported by: The University of Chile, Project FONDECYT $\mathrm{n}^{\circ}$ 1970732, the Ministerio de Ciencia y Tecnología de España. Project: 'Numerical simulation of the seismic behavior of structures with energy dissipation systems'. Contract $n^{\circ}$ : BIA2003-08700-C03-02 and the Integrated R\&D Project of the EC "LessLoss-Risk Mitigation for Earthquakes and Landslides" partially funded by the European Commission, Directorate General of Research under the Contract $n^{\circ}$ GOCECT-2003-505448.

\section{References}

AASHTO [1991] Guide Specifications for Base Isolation Design, A. Assoc. of State Hwy. and Transp. Officials, Washington, D.C.

Ahmadi, H. R. and Muhr, A. H. [1997] "Modelling dynamic properties of filled rubber," Plastics, Rubber and Composites Processing and Applications, 26(10), 451-461.

Asano, M., Masahiko, H., and Yamamoto, M. [2001] "The experimental study on viscoelastic material dampers and the formulation of analytical Model," Proceedings of the 12th World Conference on Earthquake Engineering, Paper n ${ }^{\circ} 1535$. 
Barbat, A. H. and Bozzo, L. M. [1997] "Seismic analysis of base isolated buildings," Archives of Computational Methods in Engineering, 4(2), 153-192.

Barbat, A. H., Oller, S. Oñate, E., and Hanganu, A. [1997] "Viscous Damage Model for Timoshenko Beam Structures," International Journal of Solids Structures, 34, 3953-3976.

Belytschko, T. Liu, W. K., and Moran, B. [2000] Nonlinear Finite Elements for Continua and Structures, John Wiley \& Sons, Inc., New York.

Bixio, A. R., Dolce, M., Nigro, D., Ponzo, F. C., Braga, F. and Nicoletti, M. [2001] "Repeatable dynamic release tests on a base-isolated building" Journal of Earthquake Engineering, 5(3), 369-393.

Calvi, G. M. and Pavese, A. [1997] "Conceptual design of isolation systems for bridge structures" Journal of Earthquake Engineering 1(1), 193-218.

Chopra, A. [2000] Dynamics of Structures: Theory and Applications to Earthquake Engineering, 2nd ed., Prentice Hall, Inc. Upper Saddle River, New Jersey.

Dusi, A., Bettinali, F., Rebecchi, V., and Bonacina, G. [1999] "High damping laminated rubber bearings (HDLRBs): A simplified non linear model with exponential constitutive law. Model description and validation through experimental activities," ECCMR '99-First European Conference on Constitutive Models for Rubber. Vienna, Austria.

Kojima, H. and Yoshihide, Y. [1990] "Performance, durability of high damping rubber bearings for earthquake protection," Rubber World, 202(4).

Hwang, J. S. and Ku, S. W. [1997] "Analytical modelling of high damping rubber bearings," Journal of Structural Engineering, 123(8).

Kelly, J. [1988] Earthquake Resistant Design with Rubber, Department of Civil Engineering, University of Chile (Textbook).

Mata, P. and Boroschek, R., [2001] "Caracterización mecánica de goma de alto amortiguamiento para el desarrollo de disipadores de energía," $2^{\circ}$ Congreso Iberoamericano de Ingeniería Sísmica, 10-16 Oct, Madrid, Spain.

Mata, P., Boroschek, R., and Barbat, A. H. [2004] "Analytical model for high damping elastomers applied to energy dissipating devices. Numerical study and experimental validation," Proceedings of the Third European Conference on Structural Control, 3ECSC, Vienna, Austria.

Moroni, M., Sarrazín, R., and Boroschek, R. [1988] "Experiments on a base isolated buildings in Santiago, Chile," Engineering Structures, 20(8), 720-725.

Naeim, F. and Kelly, J. [1999] "Design of Seismic Isolated Structures. From Theory to Practice," John Wiley \& Sons, Inc., New York.

Ponce León, E., Vera, V., Moroni, O., Boroschek, R., and Sarrazín, M. [1997] "Desarrollo y Ensayo de Disipadores de Energía por Deformación de Material Viscoso," Séptimas Jornadas Chilenas de Sismología e Ingeniería Antisísmica y Primer Congreso Iberoamericano de Sismología, Noviembre, La Serena, Chile.

Salomón, O., Oller, S., and Barbat, A. [1999] "Modelo numérico de elastómeros multi-fase y su aplicación a estructuras con aislamiento sísmico," Monografía CIMNE IS-36, Technical University of Catalonia, UPC.

Salomon, O., Oller, S., and Barbat, A. H. [1999] "Finite element analysis of base isolated buildings subjected to earthquake loads," International Journal for Numerical Methods in Engineering, 46, 1741-1761.

SAP [2004] CSI Analysis Reference Manual for SAP2000, ETABS and SAFE, Computer and Structures Inc., Berkeley, California.

Sarrazín, M. Moroni, M., and Boroschek, R. [1993] "Experiments on a Base Isolated Confined Masonry Buildings," Proceedings ATC, 17-1 Seminar on Seismic Isolation, Passive Energy Dissipation, and Active Control, Vol. 1, San Francisco.

Sommer, J. G. [1989] "Effect of shape and other design factors on rubber behaviour," Rubber World, 201(3).

Soong, T. T. and Dargush, G. F. [1997] Passive Energy Dissipation System in Structural Engineering, John Wiley \& Sons, Inc., New York.

Sues, R. H., Mau, S. T., and Wen, Y. K. [1988] "System identification of degrading hysteretic restoring forces," Journal of Engineering Mechanics 114( 5). 
Wen, Y. K. [1976] "Method for random vibration of hysteretic systems," Journal of the Engineering Mechanics Division. ASCE 102(2), 249-263.

Wilson, E. L. [1998] "Three dimensional static and dynamic analysis of structures, a physical approach with emphasis on earthquake engineering," Computers and Structures, Inc. Berkeley, California.

Valles, R. E., Reinhorn, M., Kunnath, S. K., Li, C., and Madan, A. [1996] "IDARC-2D, A program for inelastic Damage Analysis of Buildings," Technical Report NCEER 96-00-10. National Center for Earthquake Engineering Research. 